\title{
De novo sequencing of the Hypericum perforatum L. flower transcriptome to identify potential genes that are related to plant reproduction sensu lato
}

\author{
Giulio Galla', Heiko Vogel${ }^{2}$, Timothy F Sharbel ${ }^{3}$ and Gianni Barcaccia ${ }^{1 *}$
}

\begin{abstract}
Background: St. John's wort (Hypericum perforatum L.) is a medicinal plant that produces important metabolites with antidepressant and anticancer activities. Recently gained biological information has shown that this species is also an attractive model system for the study of a naturally occurring form of asexual reproduction called apomixis, which allows cloning plants through seeds. In aposporic gametogenesis, one or multiple somatic cells belonging to the ovule nucellus change their fate by dividing mitotically and developing functionally unreduced embryo sacs by mimicking sexual gametogenesis. Although the introduction of apomixis into agronomically important crops could have revolutionary implications for plant breeding, the genetic control of this mechanism of seed formation is still not well understood for most of the model species investigated so far. We used Roche 454 technology to sequence the entire $\mathrm{H}$. perforatum flower transcriptome of whole flower buds and single flower verticils collected from obligately sexual and unrelated highly or facultatively apomictic genotypes, which enabled us to identify RNAs that are likely exclusive to flower organs (i.e., sepals, petals, stamens and carpels) or reproductive strategies (i.e., sexual vs. apomictic).
\end{abstract}

Results: Here we sequenced and annotated the flower transcriptome of $H$. perforatum with particular reference to reproductive organs and processes. In particular, in our study we characterized approximately 37,000 transcripts found expressed in male and/or female reproductive organs, including tissues or cells of sexual and apomictic flower buds. Ontological annotation was applied to identify major biological processes and molecular functions involved in flower development and plant reproduction. Starting from this dataset, we were able to recover and annotate a large number of transcripts related to meiosis, gametophyte/gamete formation, and embryogenesis, as well as genes that are exclusively or preferentially expressed in sexual or apomictic libraries. Real-Time RT-qPCR assays on pistils and anthers collected at different developmental stages from accessions showing alternative modes of reproduction were used to identify potential genes that are related to plant reproduction sensu lato in H. perforatum.

Conclusions: Our approach of sequencing flowers from two fully obligate sexual genotypes and two unrelated highly apomictic genotypes, in addition to different flower parts dissected from a facultatively apomictic accession, enabled us to analyze the complexity of the flower transcriptome according to its main reproductive organs as well as for alternative reproductive behaviors. Both annotation and expression data provided original results supporting the hypothesis that apomixis in $\mathrm{H}$. perforatum relies upon spatial or temporal mis-expression of genes acting during female sexual reproduction. The present analyses aim to pave the way toward a better understanding of the molecular basis of flower development and plant reproduction, by identifying genes or RNAs that may differentiate or regulate the sexual and apomictic reproductive pathways in H. perforatum.

Keywords: Hypericum perforatum, Flower, Reproductive organs, Apomixis, Apospory

\footnotetext{
* Correspondence: gianni.barcaccia@unipd.it

'Laboratory of Plant Genetics and Genomics, DAFNAE - University of Padova,

Campus of Agripolis, Viale dell'Università 16, 35020 Legnaro, Italy

Full list of author information is available at the end of the article
}

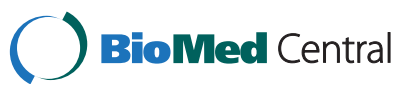

(c) 2015 Galla et al.; licensee BioMed Central. This is an Open Access article distributed under the terms of the Creative Commons Attribution License (http://creativecommons.org/licenses/by/4.0), which permits unrestricted use, distribution, and reproduction in any medium, provided the original work is properly credited. The Creative Commons Public Domain Dedication waiver (http://creativecommons.org/publicdomain/zero/1.0/) applies to the data made available in this article, unless otherwise stated. 


\section{Background}

Reproduction is the highest priority of all living things, and involves a complex combination of processes whose variability between species has long puzzled evolutionary biologists. In angiosperms, reproduction for the most part culminates in the formation of male and female gametophytes, whose union leads to a new sporophyte generation that will begin from seeds. In the flower, male and female sporogenesis and gametogenesis generate two types of gametes that are either enclosed in an embryo sac (egg cell), or pollen grain (sperm).

Despite the enormous complexity and heterogeneity of flower morphology existing within angiosperms, a number of recent publications focusing on the genes involved in flower development suggested that the biological basis of flower development is actually highly conserved among plants [1]. Accordingly, the genetic and epigenetic bases of gamete formation have been studied in model species, such as Arabidopsis, snapdragon and maize, using a number of different approaches. The production and characterization of mutants defective for their ability to set seeds [2,3], in addition to expression studies [4], led to the identification of several genes that are potentially or effectively associated with sporogenesis and gametogenesis [5]. A number of mutations affecting the normal progression of meiosis [6] and gametophyte development [2,7], as well as double fertilization and seed development [8], have been studied in great detail (reviewed by [5]). Interconnections between the ovule and the developing embryo sac have emerged in a number of studies in which sporophytic mutations directly affecting gametophytic development were studied [9-11]. More recently, the mutation of genes involved in the production and action of sRNAs in the ovule have been shown to have dramatic effects on the development of the embryo sac $[12,13]$, thus supporting the idea that sRNA-related mechanisms residing in the sporophyte are critical for cell-fate determination in the ovule and the formation of gametes [13-15].

Increasing interest has been focused on Hypericum perforatum for the study of apomixis, a naturally occurring form of asexual reproduction whereby progeny inherit the entire maternal genome through the seed [16-20]. Hypericum spp. (1.3 pg/2C, equal to $\sim 630 \mathrm{Mb})$ have $x=8$ chromosomes: individuals are mainly tetraploid with $2 n=32$, but diploid and hexaploid individuals have been found. $H$. perforatum is an invasive perennial herb that is widely distributed in a variety of habitats and is regarded as a serious weed in many countries $[21,22]$. Several compounds produced by Hypericum species have stimulated the interest of the scientific community for their biological activity [23], and $H$. perforatum has been studied for the identification of potential genes involved in the biosynthesis of active metabolites
[24]. From the reproductive point of view, $H$. perforatum reproduces via aposporic apomixis, a gametophytic variant (according to Nogler, [25]) whereby the alternative differentiation of a somatic cell gives rise to a functional, unreduced embryo sac. In principle, aposporic initial cells in apomictic plants are somatic cells belonging to the nucellus, which change their fate by being able to mitotically divide and develop functional embryo sacs through mimicking sexual gametogenesis development $[19,26]$. The mode of reproduction in $H$. perforatum is highly dynamic, and biotypes span from almost complete sexuality to nearly obligate apomixis. In particular, apomixis is mostly found in tetraploid individuals and is characterized by complete penetrance and variable levels of expressivity, ranging from $20 \%$ to $97 \%$ (for review, see Pupilli and Barcaccia, [27] and ref. therein). The occurrence of diploid and hexaploid individuals reflects a dynamic reproductive system because haploidization and polyploidization are mediated by parthenogenesis of meiotic egg cells and fertilization of aposporic egg cells, respectively $[17,26]$. As with other asexual plant complexes, apomixis and hybridization are closely linked in $H$. perforatum [28], and interestingly the dosage of genetic factors has been proposed to influence the penetrance of apomixis, as tetraploid and hexaploid genotypes tend to be more apomictic and sexual respectively, regardless of geographic origin [29]. These observations are in agreement with the hypothesis that apomixis might relies upon spatial or temporal mis-expression of genes acting during female sexual reproduction [30].

Hypericum perforatum is considered an attractive model system for the study of apomixis because it is characterized by a relatively small genome size, the availability of morphologically distinct ecotypes, self-compatibility and easy cross-ability, high degree of molecular polymorphisms, along with a versatile mode of reproduction, a relatively short generation time and an abundant seed set [26,31]. Genotypes that produce embryos either from aposporic fertilized egg cells or from parthenogenesis of meiotically reduced egg cells have been identified, suggesting that apospory and parthenogenesis may be developmentally uncoupled [17]. It is now well known that parthenogenic capacity is preferentially expressed by aposporic egg cells (or, alternatively, non-parthenogenic behavior is frequently associated with meiotic egg cells). Nevertheless, aposporic egg cells can frequently occur in non-parthenogenic individuals, and parthenogenic development of meiotic egg cells can also take place. Genotypes that almost exclusively express only one component of apomixis while suppressing the other support the hypothesis that two distinct genetic factors control apospory and parthenogenesis in this species (for review, see Barcaccia et al., [27] and ref. therein). Recently, the genetic basis of apomixis in $H$. perforatum was uncovered by mapping 
and then sequencing a locus (designated HAPPY for Hypericum APOSPORY) associated with apospory, demonstrating that distinct genetic factors are associated apospory and parthenogenesis in this species [18].

Recently, next generation sequencing (NGS) technologies have been used to investigate gene expression changes associated with sperm development [32], differentiation of the megaspore mother cell [33] and specific cell types of the embryo sac [34] in Arabidopsis. In an alternative approach, high-quality single nucleotide polymorphisms (SNPs) were mined from NGS libraries sequenced from a number of sexual and apomictic Ranunculus genotypes in order to elucidate the origin and evolution of apomixis [35]. Taken together, the availability of reference DNA and RNA datasets are increasing the ease at which complex phenotypes and processes in non-model organisms can be analyzed. Here we sequence and annotate the flower transcriptome of $H$. perforatum with particular reference to processes related to reproduction. The annotation and comparative investigation of the flower transcriptome is a critical step for better understanding the genetic control of apomixis, and provides valuable information on gametophyte determination and gamete formation in Hypericum.

\section{Results}

\section{De novo assembly and annotation of the Hypericum flower transcriptome}

We analyzed two sexual and three apomictic accessions (two obligate and one unrelated facultative apomictic accession characterized by $24 \%$ apomictic seed development; Table 1; Figure 1, panel A). Flower buds and flower organs were collected at different developmental stages corresponding to Arabidopsis flower stages 1-12 and spanning meiosis and gametogenesis processes (Figure 1, panels B-G, with particular reference to the female gametophyte).
Samples were collected to include the entire developmental pathway of gamete production, whose critical steps in sexual ovules include megaspore mother cell differentiation (Figure 1, panel B), its meiotic division to form a functional megaspore (Figure 1, panel C) and mitotic divisions giving rise to a functional eight-nucleate embryo sac with an egg cell apparatus (Figure 1, F-G). Conversely, aposporic ovules (Figure 1, panel I) are frequently characterized by a failure of the meiotic program and differentiation of one or multiple unreduced embryo sacs from the somatic cells (aposporic initials) of the ovule (Figure 1, panels D-E).

All full-length enriched cDNA libraries were successfully normalized (data not shown) in order to avoid over representation of the most commonly transcribed genes and to maximize sequence diversity. Roche 454 sequencing of two full plates yielded a total of 1.47 million sequences, and an average number of sequenced reads from each library of 177,822 (Additional file 1: Table S1).

High quality sequences from all libraries were pooled and assembled to create a single global reference transcriptome, and yielded 33,860 isotigs with an average length of 1,002 bp and an N50 of 1,184 bp (Table 2). Following the assembly procedure, the quote of singletons was equal to $2 \%$ (Table 2 ). As a result, the assembly pipeline produced 60,428 assembled sequences - from now onward defined as unigenes - that were composed of 33,860 isotigs (accounting for 98\% of sequenced reads) and 26,568 singletons (Table 2).

BLASTX analysis aligned approximately $61 \%$ of the Hypericum unigenes to a protein present in the nonredundant $(\mathrm{nr})$ database $(\mathrm{n}=36,988$; E-value $\leq 1$.E-06; Table 3). The remaining $39 \%$ of assembled unigenes $(\mathrm{n}=23,455$; Table 3$)$ did not yield significant matches at this level of stringency. The relative contribution of assembled and non-assembled reads to the annotation of

Table 1 Information on the $\boldsymbol{H}$. perforatum accessions used for flower transcriptome analyses

\begin{tabular}{|c|c|c|c|c|c|c|}
\hline Accession & Description & Genealogy & Origin & Ploidy & Apospory & Reproduction \\
\hline $13 \mathrm{EU}^{* 0}$ & Experimental population & $4(\mathrm{~F} 12 \times \mathrm{An}) 1 / 4$ & IPK-Gatersleben (D) & $2 n=4 x$ & $<4 \%$ & Sexual \\
\hline $20 E U^{\circ}$ & Experimental population & $4(\mathrm{~F} 12 \times \mathrm{An}) 1 / 6$ & IPK-Gatersleben (D) & $2 n=4 x$ & $<4 \%$ & Sexual \\
\hline $36 \mathrm{EU}^{*}$ & Experimental population & $4(\mathrm{~F} 12 \times \mathrm{An}) 1 / 9$ & IPK-Gatersleben (D) & $2 n=4 x$ & $<4 \%$ & Sexual \\
\hline $141 \mathrm{EU}^{\circ}$ & Experimental population & $4(\mathrm{~F} 12 \times \mathrm{No}) 1 \mathrm{a} / 39$ & IPK-Gatersleben (D) & $2 n=4 x$ & $<4 \%$ & Sexual \\
\hline $\mathrm{Hp} 4 / 13^{*}$ & Wild population & n.a. & Feltre (I) & $2 n=4 x$ & $24 \%$ & Facultative apomictic \\
\hline $39 \mathrm{EU}^{*}$ & Experimental population & $4(\mathrm{~F} 12 \times \mathrm{No}) 1 \mathrm{a} / 1$ & IPK-Gatersleben (D) & $2 n=4 x$ & $>96 \%$ & Obligate apomictic \\
\hline $222 \mathrm{EU}^{\circ}$ & Experimental population & $4(\mathrm{R} 1 \mathrm{C} 2 \times \mathrm{Si}) 1 \mathrm{C} / 2$ & IPK-Gatersleben (D) & $2 n=4 x$ & $>96 \%$ & Obligate apomictic \\
\hline 1886 US $^{\circ}$ & Wild population & n.a. & Iron Mountain (USA) & $2 n=4 x$ & $>96 \%$ & Obligate apomictic \\
\hline 1973US* & Wild population & n.a. & Tecumseh (USA) & $2 n=4 x$ & $>96 \%$ & Obligate apomictic \\
\hline $3348 \mathrm{EU}^{\circ}$ & Wild population & n.a. & Hamburg (D) & $2 n=4 x$ & $>96 \%$ & Obligate apomictic \\
\hline
\end{tabular}

For each plant accession, the origin, ploidy and degree of apomixis are indicated. Accessions marked with * were used for 454 sequencing, while those with ${ }^{\circ}$ were used for Real-Time RT-qPCR. Apospory expressed as percentage was determined by flow cytometric screening of 48 single seeds. For details on the origin and composition of experimental populations please refer to [18]. 


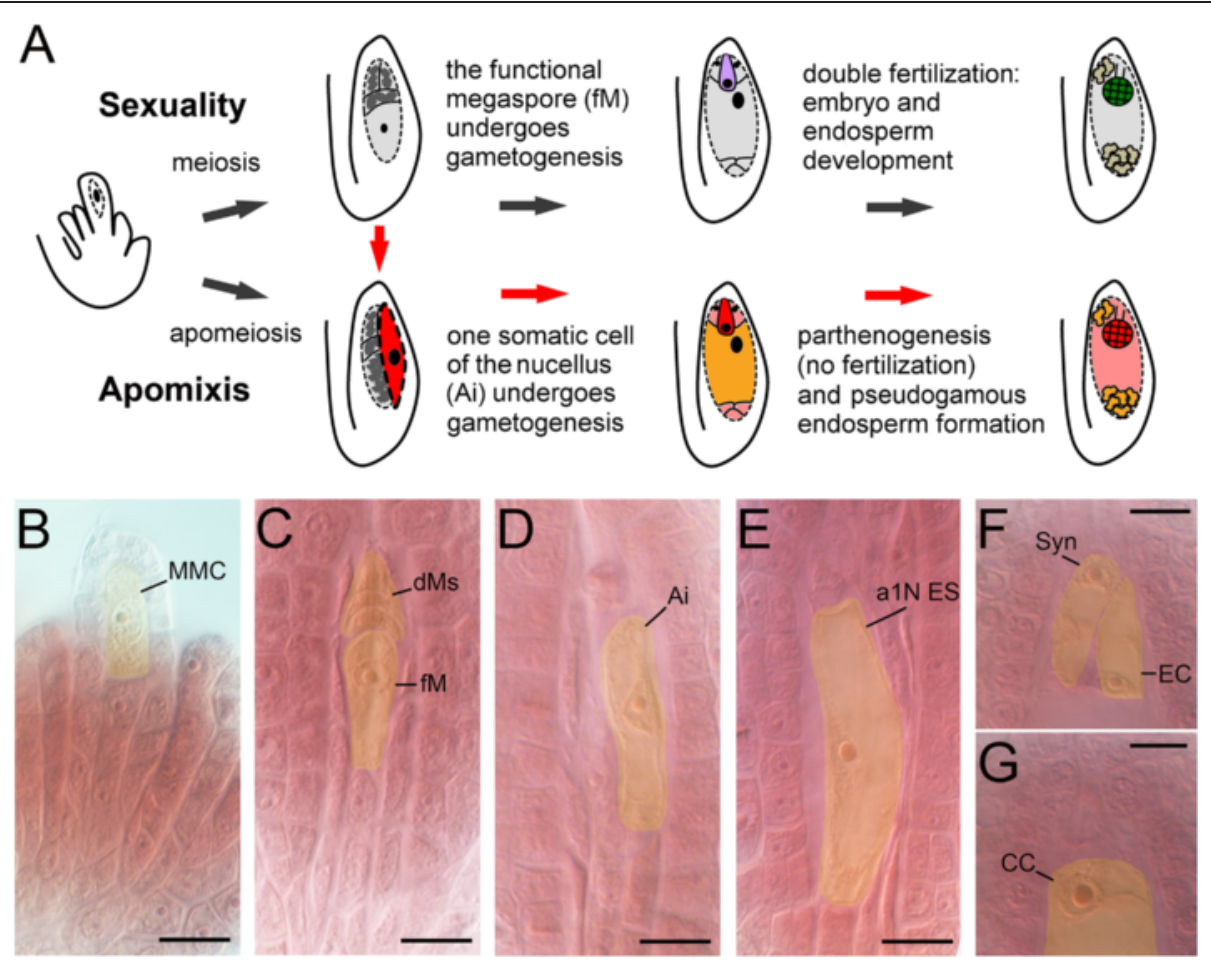

Figure 1 Developmental stages of $H$. perforatum ovules sampled and analyzed in this research. A: Schematic representation of key aspects of sexual and apomictic reproductive pathways in H. perforatum and key developmental stages. B-G: Ovules at developmental stages spanning meiosis, aposporic initial formation and development, and gametogenesis. B: megaspore mother cell (MMC); C: tetrad showing a functional megaspore (fM) along with three degenerating megaspores (dM); $\mathbf{D}$ : aposporic initial (Ai) cell; E: enlarged aposporic cell equivalent to an aposporic one-nucleate embryo sac (a1N ES); F: oangic apparatus showing in focus one synergid (Syn) and the egg cell (EC); G: detail of a central cell (CC) from a mature embryo sac. In each photograph the yellow area mark a given germinal cell type (each bar is $8 \mu \mathrm{m}$ ).

the Hypericum flower unigenes was also computed with a BLASTX-based procedure (see Additional file 1: Table S2).

As shown in Table 3, 90\% of the best hits produced by BLASTX were concentrated in 10 species, whereas the remaining $10 \%$ of BLASTX hits were distributed over 632 taxonomic entities. Roughly $86 \%$ of the most significant alignments were made with proteins deduced from Ricinus communis (35\%), Populus trichocarpa (32\%), Vitis vinifera (12\%), Glycine $\max (5 \%)$ and Medicago truncatula (2\%; Table 3).

\begin{tabular}{|c|c|}
\hline Assembled reads & 1,248,201 (87.7\%) \\
\hline Number of Isotigs & 33,860 \\
\hline Singletons & $26,568(1.9 \%)$ \\
\hline Repeat/Outlier/Tooshort & $76,514(5.6 \%)$ \\
\hline Average size of Isotigs & 1,002 \\
\hline N50 Isotig size & 1,184 \\
\hline Larger Isotig & 1,4574 \\
\hline Average number of Reads/lsotigs & 40.20 \\
\hline
\end{tabular}

The reference assembly was built starting from the pool of reads sequenced from each library.
Table 3 Number of hits resulting from BLASTX analyses of the assembled Hypericum sequences

\begin{tabular}{|c|c|c|}
\hline Species & Hits & BLAST (\%) \\
\hline Ricinus communis & 12,950 & $35.0 \%$ \\
\hline Populus trichocarpa & 11,887 & $32.1 \%$ \\
\hline Vitis vinifera & 4,329 & $11.7 \%$ \\
\hline Glycine max & 1,788 & $4.8 \%$ \\
\hline Medicago truncatula & 734 & $2.0 \%$ \\
\hline Arabidopsis thaliana & 660 & $1.8 \%$ \\
\hline Arabidopsis lyrata & 384 & $1.0 \%$ \\
\hline Lotus japonicas & 223 & $0.6 \%$ \\
\hline Jatropha curcas & 186 & $0.5 \%$ \\
\hline Hypericum perforatum & 183 & $0.5 \%$ \\
\hline Others (632 Taxonomic IDs) & 3,664 & $9.9 \%$ \\
\hline Sequences with Blast Results $\left({ }^{*}\right)$ & 36,988 & $61.2 \%$ \\
\hline Sequences without Blast Results $\left(^{*}\right)$ & 23,455 & $38.8 \%$ \\
\hline
\end{tabular}

BLAST (\%) is referred to the total number of sequences having at least one BLAST hit, with reference to the whole set of assembled sequences. 
Alignment of unigenes on the HAPPY locus and the use of this resource for the identification of allele/splice variants Screening for the identification of putative homologs in the nr database produced a number of matches with proteins encoded by genes located in the BAC clone HM061166.1 (see Additional file 1: Table S3) containing the genetic loci associated with apospory in $H$. perforatum (Figure 2, panel A). BLASTN searches of all 60,428 unigenes over the sequence of the BAC clone produced 17 relevant matches, 9 of which aligned to multiple unigenes (see Additional file 1: Table S3). Overall sequence identity shared by unigenes and the corresponding regions of HM061166.1 was on average 95\%. Moreover, BLASTN searches did not produce reliable matches for the retrotransposons located in the BAC clone. The fact that the truncated genes ARI-T, HK2 and GH2 did not match none of the unigenes from our collection (Figure 2, panel A) may indicate that these genes were not expressed or were expressed at levels below our detection threshold. Alternatively, divergence between the genetic loci of the investigated plants (Figure 2, panel A) may have hampered the production of BLAST matches (see also [18]).

BLASTX matches from the $\mathrm{nr}$ protein database (Additional file 1: Table S3) also revealed possible synteny

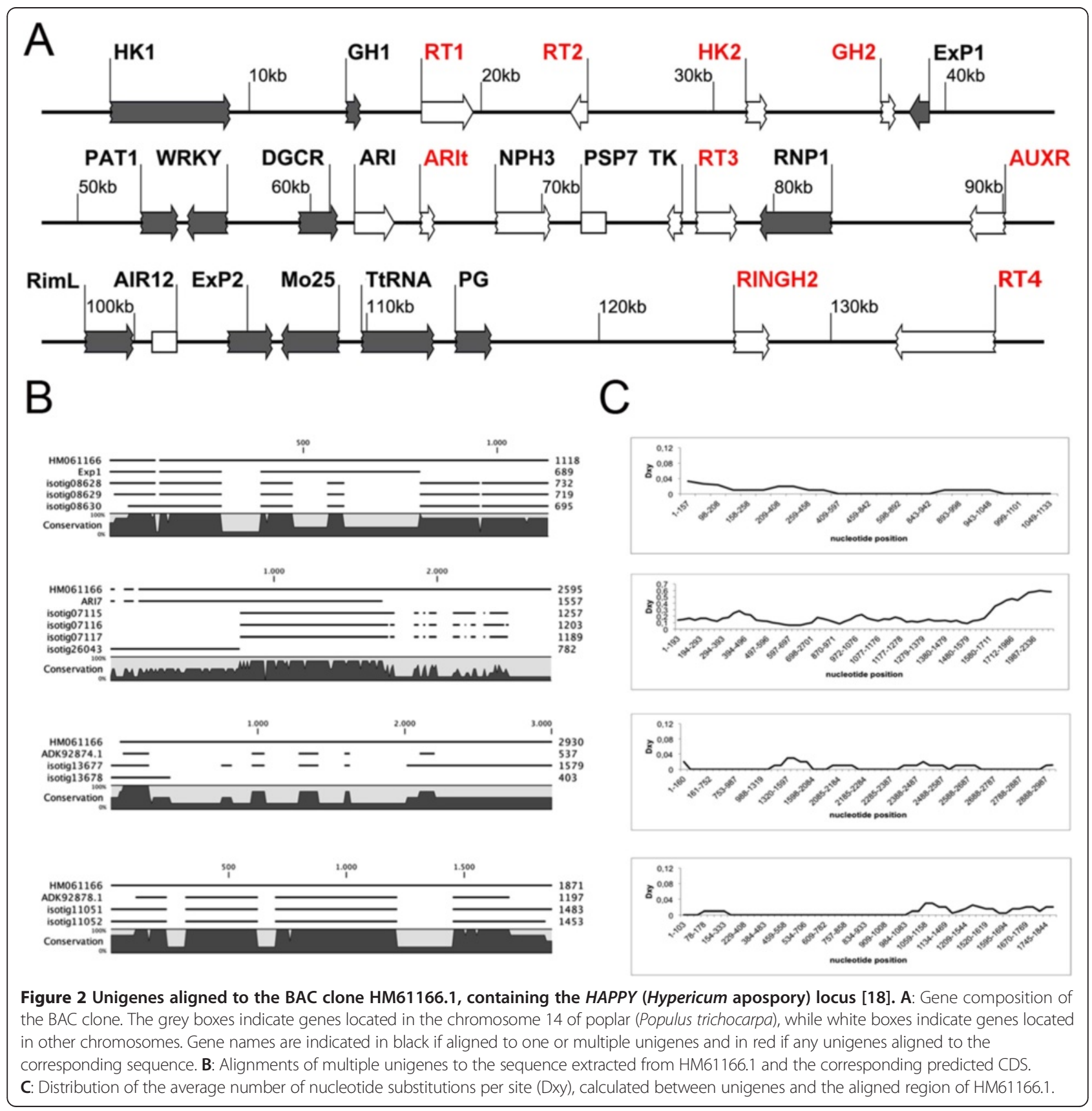


between the Hypericum sequence HM061166.1 and the chromosomal region located between the loci POPTR_ 0014s16260.1 and POPTR_0014s15970.1 of poplar (Populus trichocarpa). One exception to this was the region containing ARI and a number of other genes, which had truncated forms of ARI-T, NPH3 and TK that are not located in the same chromosome (LG14) in poplar (Additional file 1: Table S3; Figure 2). Positive matches of flower transcripts with nucleotide sequences of HM061166.1 further suggested the presence of some additional coding regions in the BAC clone, including PSP7 and AIR12.

Since distinct unigenes are the product of different assembly output, it is possible that the finding of multiple unigenes matching the same BAC portion reflects the existence of duplicated genes (i.e., paralogs) or allelic variation existing in our pool of genotypes. To test this hypothesis we selected 9 regions of the BAC clone HM061166.1 that shared high similarity with multiple unigenes (Figure 2; Table 4), aligned the sequences in multiple alignments and analyzed the nucleotide diversity existing between unigenes and reference sequence extracted from HM061166.1. As external controls, the same statistics were made for the three single copy regions ITS or internal transcribed spacers, matK and ndhF (Table 4). Nucleotide diversity displayed by unigenes when compared to their reference sequences extracted from HM061166.1 was an average 0.044 \pm 0.062 (within unigenes: $0.025 \pm 0.026$ ). Conversely, the nucleotide diversity estimated for the control regions ITS, matK and ndhF was much lower and averaging $0.01 \pm$ 0.01 (Table 4). The average nucleotide diversity measured in the BAC regions surrounding ARI7-like was as low as $0.013 \pm 0.012$ (within unigenes: $0.013 \pm 0.016$ ). Nucleotide diversity for sequences aligning to the ARI7like gene was equal to 0.170 (within unigenes: 0.080). For a number of unigenes, the nucleotide diversity shared with genes encoding for WRKY-like protein,

Table 4 Diversity statistics for the unigenes aligning to the Hypericum BAC clone HM06166

\begin{tabular}{|c|c|c|c|c|c|c|c|}
\hline Gene product & Accession & Unigenes & S/AL & $\mathrm{Pi} / \mathrm{AL}$ & $S / U$ & $\mathrm{Pi} / \mathrm{U}$ & $\mathrm{Hn} / \mathrm{ALU}$ \\
\hline \multirow[t]{3}{*}{ Predicted protein } & XP_002883670.1 & isotig08628 & & & 7 & 0.007 & 4 \\
\hline & & isotig08629 & & & & & \\
\hline & & isotig08630 & & & & & \\
\hline \multirow[t]{2}{*}{ WRKY-like protein } & ADK92866.1 & isotig10799 & 4 & 0.000 & 16 & 0.010 & 3 \\
\hline & & isotig10800 & 11 & 0.010 & & & \\
\hline \multirow[t]{2}{*}{ DGCR-like protein } & ADK92867.1 & isotig04455 & 9 & 0.010 & 21 & 0.022 & 3 \\
\hline & & isotig04458 & 24 & 0.030 & & & \\
\hline \multirow[t]{4}{*}{ ARI7-like } & XP_002310443.1 & isotig26043 & 98 & 0.160 & 139 & 0.081 & 4 \\
\hline & & isotig07115 & 126 & 0.170 & & & \\
\hline & & isotig07116 & 121 & 0.160 & & & \\
\hline & & isotig07117 & 119 & 0.150 & & & \\
\hline \multirow[t]{2}{*}{ Thymidine kinase (TK) } & ADK92870.1 & isotig09624 & 7 & 0.010 & 7 & 0.008 & 3 \\
\hline & & isotig09625 & 7 & 0.010 & & & \\
\hline \multirow[t]{3}{*}{ HnRNP } & ADK92872.1 & isotig31453 & 2 & 0.020 & 26 & 0.037 & 3 \\
\hline & & isotig11295 & 3 & 0.000 & & & \\
\hline & & isotig11296 & 20 & 0.030 & & & \\
\hline \multirow[t]{2}{*}{ RimL/acetyltransferase-domain protein } & ADK92874.1 & isotig13677 & 4 & 0.010 & 0 & 0.000 & 2 \\
\hline & & isotig13678 & 0 & 0.000 & & & \\
\hline \multirow[t]{2}{*}{ Mo25-like protein } & ADK92876.2 & isotig05993 & 34 & 0.030 & 43 & 0.031 & 3 \\
\hline & & isotig05994 & 36 & 0.030 & & & \\
\hline \multirow[t]{2}{*}{ Polygalacturonase (PG) } & ADK92878.1 & isotig11051 & 3 & 0.000 & 17 & 0.012 & 3 \\
\hline & & isotig11052 & 11 & 0.010 & & & \\
\hline ITS (nuclear)** & & contig00041 & 1 & 0.002 & n.a. & n.a. & 2 \\
\hline
\end{tabular}

Statistics are based on multiple unigenes aligning to unique coding sequences predicted from the BAC clone HM061166, containing the HAPPY locus associated to Apospory ( $\mathrm{AL}$ ). For each coding region (Gene product), the Unigene Id, the number of polymorphic sites (S/AL) and measured nucleotide diversity (Pi/AL) are reported. The number of polymorphic sites $(\mathrm{S} / \mathrm{U})$ and nucleotide diversity $(\mathrm{Pi} / \mathrm{U})$ calculated among unigenes are also indicated. The number of haplotypes emerging from the alignment of the multiple unigenes to coding sequences predicted from the BAC clone HM061166 is indicated as Hn/ALU. **Locus not included in the BAC clone HM061166, which was used as external reference for statistics on sequence genetic diversity. Unigenes were deposited as TSA project under the accession GBXG00000000. 
HnRNP, RimL/acetyltransferase-domain protein, PG was equal to 0.00 (Table 4). Despite the low genetic diversity, the unigenes aligned to the genes: Exp1, DGCR-like protein, HnRNP, RimL/acetyltransferase-domain protein, and Mo25-like protein displayed alignment variants featuring a possible lack or insertion of sequence traits in their CDS, coding sequences (Figure 2, panel B). The distribution of the average number of nucleotide substitutions per site (Dxy) calculated between unigenes and corresponding regions of HM061166.1 showed that nucleotide substitutions were concentrated in the 3'-end of the alignments in at least $50 \%$ of investigated regions (Figure 2, panel C, see also Additional file 2: Figure S1).

The distribution of sequences between single libraries

Of the 60,428 unigenes composing our reference assembly, and identified from different flowers and flower parts, 33,860 sequences were assembled with reads derived from one or multiple libraries. Calculation of the number of raw reads assembled in each unigene from each library allowed us to estimate the presence of each unigene in each sequenced library. These data were used to infer the overlaps existing between the two sequencing reactions (sequencing plates) and independent libraries (i.e., 4 libraries for each sequencing plate). About $92 \%$ of the assembled unigenes were composed of sequences shared between the two independent sequencing experiments (Figure 3, panel A). As much as $86 \%$ of unigenes were identified from the flowers of at least three sexual or apomictic individuals (Figure 3, panel B). Similarly, 78\% of unigenes were shared by at least three libraries made from the different flower parts. Taken together these results indicate that most transcripts were detectable at the flowertranscriptome level, regardless of reproductive mode or specific flower organ, while numbers of transcripts detectable in single libraries were low (Figure 3, panels B-C).

The existence and abundance of overlaps between the libraries derived from flowers collected from sexual and obligate apomictic accessions were also measured (Figure 3, panel B). A total of 552 (1.7\% of assembled unigenes) transcripts were present in both apomictic libraries, but were not found in the sexual libraries. Moreover, a total of 673 sequences $(2.0 \%$ of assembled unigenes) were observed in the two sexual samples, but not in the apomictic samples.

When we considered the different flower parts collected from a facultative apomictic accession (characterized by $24 \%$ apospory), and hence showing either apomictic or sexual reproduction, we could identify 616 unigenes $(1.9 \%$ of total unigenes) exclusive to young flower buds at stages preceding micro- and mega-sporogenesis (Figure 3, panel C). Moreover, 765 and 1,178 sequences were sequenced only in carpels and stamens, respectively (equal to $2.4 \%$ and $3.7 \%$ of total unigenes, respectively) (Figure 3 ,

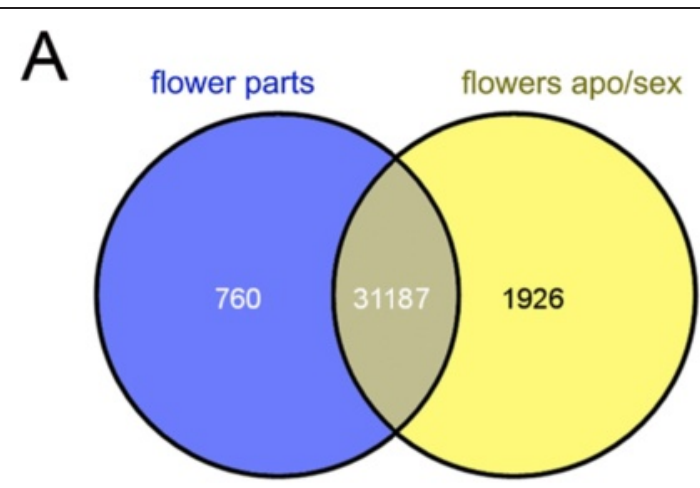

B

Flowers apo / sex

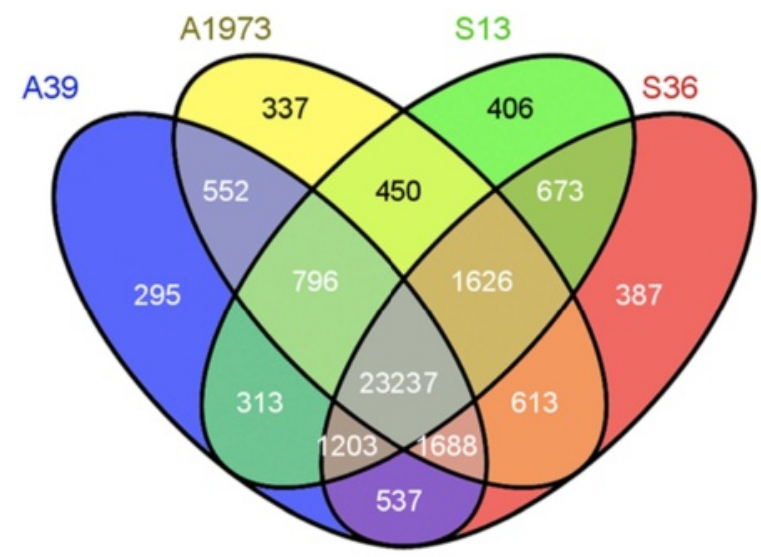

C

Flower parts

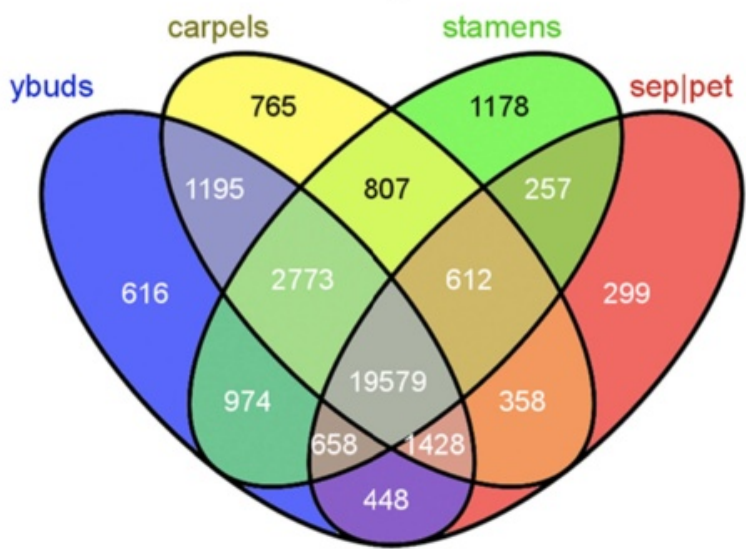

Figure 3 Statistics on the composition of libraries and distribution of unigenes. Venn diagrams that graphically represent the number of H. perforatum unigenes whose presence was shared among libraries, as indicated by the contribution of each library to the assembly. All possible intersections between libraries, as well as library specific sequences, are shown. A: Sequences shared by the two sequencing approaches; $\boldsymbol{B}$ : Sequences derived from whole flowers of two apomictic (A39, A1973) and two sexual (S13, S36) individuals; C: Sequences derived from single flower parts collected from a facultative apomictic accession. sep|pet: sepals and petals. 
panel C). The overlap between carpels and stamens consisted of 807 (2.5\%) single unigenes. Similarly, 1,195 and 974 transcripts were found in the overlapping regions of young buds with carpels and stamens, respectively.

Finally, most genes residing on the genomic region comprised in the BAC clone HM061166.1, thus surrounding the HAPPY locus, were expressed in the different flower parts as well as in both sexual and apomictic flowers analyzed in this study, as indicated by the production of multiple sequence reads matching the CDS predicted from the genomic sequence HM061166.1 (see Additional file 1: Table S4).

\section{Ontological annotation of sexual and apomictic specific sequences}

For the annotation of gene products with a possible key role in plant reproduction and seed formation, we checked the ontological annotation associated to all unigenes identified only in sexual and apomictic libraries (673 and 552 unigenes, respectively; Figure 3). Metrics concerning the full ontological annotation of the flower transcriptome are reported on Additional file 1: Table S5. Most annotations associated to unigenes identified only in sexual and apomictic libraries were shared between the two sequence collections, although some extent of variability was observed for single GO annotations (Figure 4).

Highest scores were recorded for terms associated to the sensing and response stresses and stimuli as well as terms associated to metabolism of carbohydrate, lipids and secondary compounds (Figure 4, panel A). The annotations: cell differentiation (hits: 3, GO:0030154), cell cycle (hits: 11, GO:0007049) and embryo development (hits: 5, GO:0030154) were also identified at lower extend. Few sequences identified only in the sexual libraries could be annotated as regulation of gene expression, epigenetic (hits: 1, GO:0040029), cell growth (hits: 2, GO:0016049), flower development (hits: 2, GO:0009908) and pollination (hits: 2, GO:). Likewise, cell-cell signaling (hits: 1, GO: 0007267), cellular homeostasis (hits: 3, GO:0019725), post-embryonic development (hits: 2, GO:0009791) and reproduction (hits: 2, GO:0000003) were associated to a small number of sequences specific of the apomictic libraries (Figure 4, panel A). As far as the molecular function of unigenes identified only in sexual and apomictic libraries is concerned, the most frequent annotations were DNA binding (hits: 55, GO:0003677), nucleotide binding (hits: 116, GO:0000166), kinase activity (hits: 42, GO: 0016301) and transporter activity (hits: 28, GO:0005215) (Figure 4, panel B).

\section{Identification of genes involved in flower development, gamete formation and plant reproduction}

As flower organs were sampled in this study, we expected the libraries to contain genes involved in flower development, gamete formation and reproduction. To test this hypothesis and annotate the unigenes, we created a database of 811 Arabidopsis genes whose function has been associated with plant reproduction sensu lato and used this set of data as a source of data specifically focused on reproduction (see Additional file 1: Table S6). BLAST analyses indicated that 1,674 Hypericum unigenes were putative orthologs to 632 Arabidopsis gene products (78\% of total considered genes associated to reproduction in the broad sense).

An attempt to join the annotation of reproductiverelated genes to their expression site was done by checking the distribution of sequences matching reproduction-related genes within the different flower parts. Approximately $63 \%$ of the sequences matching reproduction-related genes were shared among all organs. Of the remaining sequences, most were expressed in all flower tissues, with the exception of only the sepals and petals (Figure 5). To a lesser extent, sequences were present in carpels and young buds and stamens and young buds, followed by stamens and carpels alone (Figure 5). According to this finding, the distribution of apomicticor sexual-specific unigenes in each plant organ revealed that nearly half of the annotated unigenes belonged to three groups: i) young buds, carpels and stamens; ii) carpels and young buds; and iii) stamens and young buds. Approximately $10 \%$ of the apomictic- or sexual-specific unigenes could not be paired with any specific flower part (Figure 5).

Among the sequences annotated as reproductionrelated, we could effectively annotate numerous unigenes involved in sporogenesis, gametogenesis and embryogenesis (Table 5). NCBI and TAIR searches yielded $89 \%$ of the annotations related to meiosis, gametogenesis and embryogenesis (Table 5). Sequences derived exclusively from sexual libraries produced almost double the number of reproductive related genes derived from the apomictic libraries (223 and 118 unigenes, respectively). This difference was particularly evident for genes comprised in the two categories: "regulation of megasporogenesis" (17 and 5 unigenes, respectively) and "female gametogenesis, fertilization and seed development" (21 and 3 unigenes, respectively) (Table 5).

With this approach, we were able to identify and annotate Hypericum unigenes whose expression has been associated with phases of ovule development (e.g., INO, $B E L, A N T)$, along with transcripts whose activity is restricted or specific to cell fate in the ovule, such as in the nucellar tissue (e.g., AGO9, RDR6 and SGS3) and embryo sac (e.g., ATO, CLO, BLH1 and LIS) (Table 6). Similarly, we could associate 81 unigenes expressed in the Hypericum flower to meiosis (see Table 5). Among those, we detected and annotated a number of genes with known functions in meiosis (e.g., DMC1, ATREC8, 


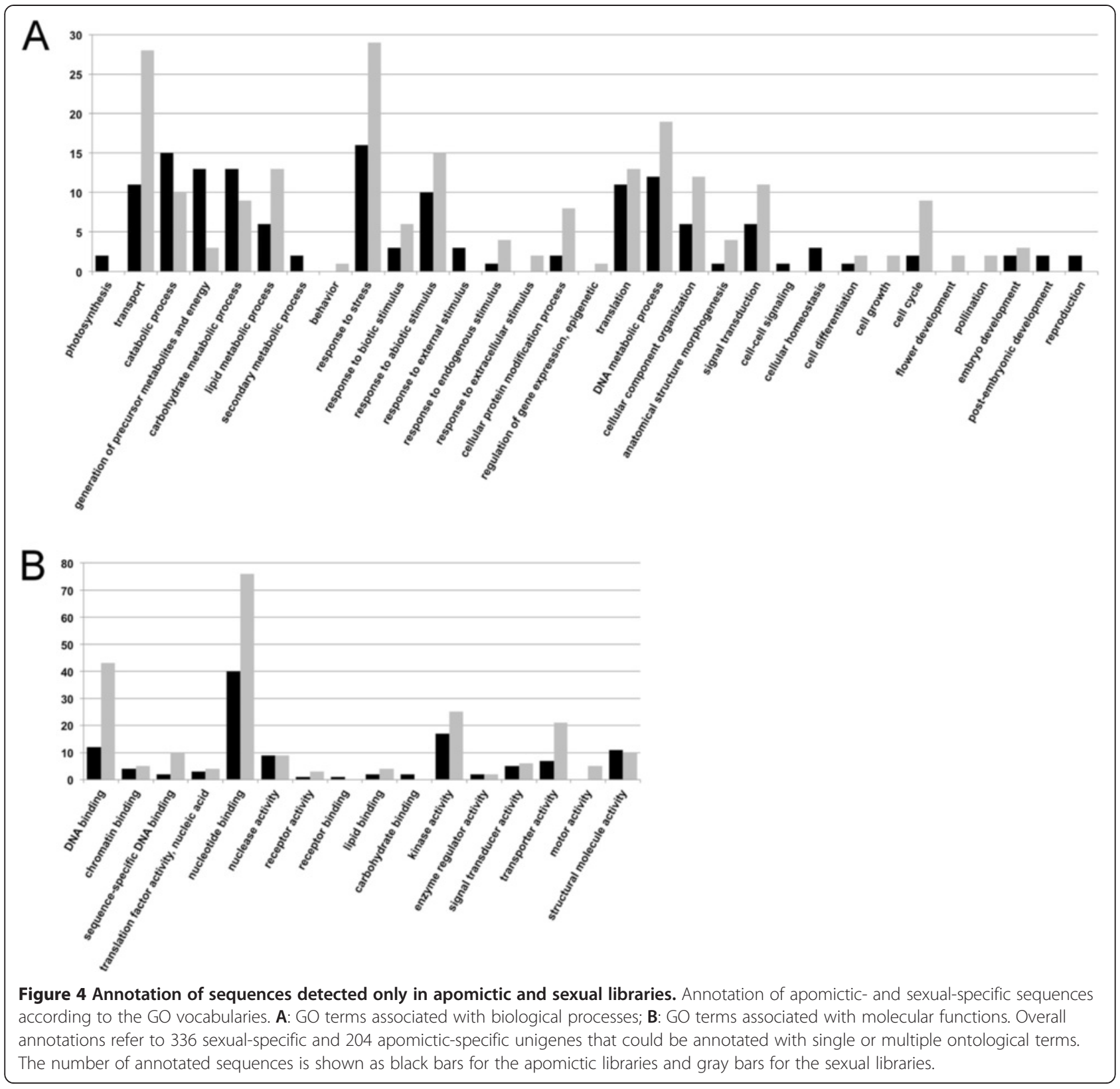

$M M D 1$ and $M S 5)$, megaspore selection and gamete formation (e.g., AGP18, AGO9, AGO5 and MYB98) (Table 6). Genes involved in the control of DNA methylation in sporophytic and gametophytic plant tissues, such as MET1 and DME, were also identified and annotated (Table 6). Similarly, 521 and 157 unigenes were linked to gametophyte and gamete development and embryogenesis, respectively (see Table 5). In these categories we could sequence and annotate a number of genes whose mutants are known to affect the embryo sac, embryo and endosperm development (e.g., AGL32, IAA32/ MEE10, dVPE, FUS2, SERK, TANMEI/EMBR2757 and ILA) (Table 6).

\section{Expression analysis of reproduction-related genes in} sexual or apomictic plant accessions

Real-Time RT-qPCR assays were used to verify whether apomictic plants are affected in the expression of genes that are expected to be involved in sexual reproductionrelated processes (Table 6). In particular, the expression of 30 unigenes was tested in pistils and stamens collected from sexual (i.e., meiotic) and apomictic (i.e., apomeiotic) accessions (Table 6; Figure 6, panel A). A sample composed by sepals and petals was also included in the analyses, as external reference.

The expression levels of genes involved in determination of cell and ovule identity was tested in apomictic 


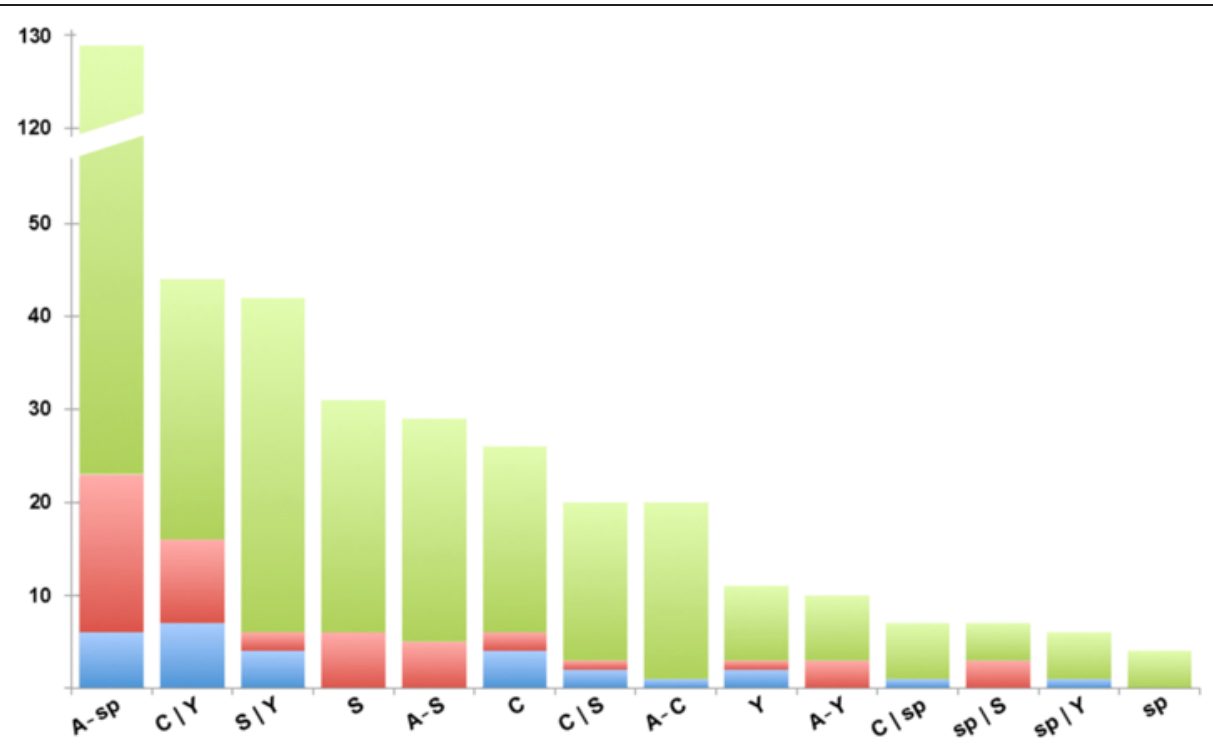

Figure 5 Abundance of sequences detected only in apomictic or sexual libraries. Distribution of reproduction-related unigenes according to the different flower parts and reproductive behaviors of H. perforatum. Legend: red, sequenced only in sexual libraries; blue, sequenced only in apomictic libraries; green, sequenced from both sexual and apomictic libraries. C: Carpels; S: Stamens; sp: Sepals/Petals; Y: Young buds; A-: present in all verticils but the one not assessed. Sequences shared by two libraries are indicated with the name of the libraries separated by "|".

and sexual pistils in order to investigate differences between meiotic and aposporic gametophyte development. In particular, we studied the expression of key genes involved in the generation and activity of small RNAs and putatively involved in the determination of cell identity in pre-meiotic and meiotic ovules (Figure 6, panel A). The expression data of HpRDR6 and HpSGS3, two genes involved in the siRNA formation in plants, did not show any differential pattern between apomictic and sexual pistils (Figure 6, panel A). Noteworthy, HpAGO9, which

Table 5 Hypericum matches to the plant reproductive database of Arabidopsis

\begin{tabular}{|c|c|c|c|c|c|c|c|}
\hline \multirow[t]{2}{*}{ Unigenes } & \multirow[t]{2}{*}{ At loci } & \multirow[t]{2}{*}{ Biological terms } & \multicolumn{2}{|l|}{ APO } & \multicolumn{2}{|l|}{ SEX } & \multirow[t]{2}{*}{ Common } \\
\hline & & & 1 repl. & 2 repl. & 1 repl. & 2 repl. & \\
\hline 20 & 9 & Cell specification & 0 & 1 & 1 & 2 & 16 \\
\hline 90 & 36 & Regulation of megasporogenesis & 3 & 2 & 16 & 1 & 68 \\
\hline 9 & 4 & Regulation of megagametogenesis & 1 & 0 & 0 & 0 & 8 \\
\hline 79 & 24 & Female gametogenesis, fertilization, seed development & 3 & 0 & 21 & 2 & 53 \\
\hline 21 & 1 & Pollen tube reception & 0 & 0 & 3 & 0 & 18 \\
\hline 26 & 10 & Seed development and size regulation & 5 & 1 & 3 & 0 & 17 \\
\hline 9 & 3 & Imprinted and imprinting related genes & 0 & 0 & 0 & 2 & 7 \\
\hline 54 & 16 & Sporophytic mutants with gametophytic defects & 1 & 2 & 3 & 3 & 45 \\
\hline 7 & 2 & Gametophytic factor & 0 & 0 & 2 & 1 & 4 \\
\hline 25 & 10 & Unfertilized embryo sac & 2 & 1 & 1 & 0 & 21 \\
\hline 93 & 36 & Maternal effect embryo arrest & 5 & 1 & 10 & 2 & 75 \\
\hline 482 & 164 & Embryo defective & 30 & 2 & 43 & 8 & 399 \\
\hline 81 & 29 & Meiosis & 8 & 1 & 15 & 6 & 51 \\
\hline 521 & 183 & Gametogenesis & 37 & 3 & 50 & 5 & 426 \\
\hline 157 & 49 & Embryogenesis & 6 & 3 & 19 & 4 & 125 \\
\hline 1,674 & 576 & Total & 101 & 17 & 187 & 36 & 1,333 \\
\hline
\end{tabular}

The reproductive database was built upon TAIR and NCBI annotations and provides information on genes involved on reproductive processes. For each biological term or process, the number of Hypericum unigenes having a significant match with entries on the reproductive database is shown (unigenes). The number of reference Arabidopsis genes matched by a Hypericum unigene are also reported (At loci). For each biological term or process, the distribution of the Hypericum unigenes among the apomictic and sexual 454 libraries are shown. APO: present only in apomictic libraries; SEX: present only in sexual libraries; Common: present in both apomictic and sexual libraries. 1 repl: detected from a single library; 2 repl: detected in either apomictic (APO) or sexual (SEX) libraries. 
Table 6 Unigenes involved in key biological steps of ovule, gamete and seed formation

\begin{tabular}{|c|c|c|c|c|c|c|c|c|c|}
\hline \multirow[t]{2}{*}{ Unigene } & \multirow[t]{2}{*}{ Gene } & \multirow[t]{2}{*}{ Biological process } & \multirow[t]{2}{*}{$\begin{array}{l}\text { DB } \\
\text { source }\end{array}$} & \multicolumn{4}{|c|}{$\begin{array}{l}\text { Libraries of } \\
\text { flower parts }\end{array}$} & \multirow{2}{*}{$\begin{array}{l}\text { Apomictic } \\
\text { libraries } \\
\text { mean } \\
\text { (st.dev.) }\end{array}$} & \multirow{2}{*}{$\begin{array}{l}\text { Sexual } \\
\text { libraries } \\
\text { mean } \\
\text { (st.dev.) }\end{array}$} \\
\hline & & & & $\mathrm{C}$ & $\mathrm{S} / \mathrm{P}$ & St & YB & & \\
\hline isotig28792 & HpANT & $\begin{array}{l}\text { Sporophytic mutants with gametophytic defects and seed } \\
\text { development and size regulation }\end{array}$ & $2 ; 8$ & 1.5 & 0.0 & 0.0 & 3.0 & $0.0(0.0)$ & $0.9(0.0)$ \\
\hline isotig03676 & HpBEL1 & Sporophytic mutants with gametophytic defects & 2 & 2.1 & 0.0 & 0.0 & 0.0 & $3.1(0.4)$ & $1.8(0.2)$ \\
\hline isotig24625 & HpINO & Sporophytic mutants with gametophytic defects & 2 & 10.5 & 0.0 & 0.0 & 0.0 & $4.4(0.0)$ & $2.1(0.3)$ \\
\hline isotig30848 & HpAGO9 & Cell specification and regulation of megasporogenesis & $1 ; 3 ; 4$ & 0.0 & 2.3 & 0.0 & 2.4 & $4.2(3.4)$ & $0.0(0.0)$ \\
\hline isotig08685 & HpRBR & Cell specification and regulation of megagametogenesis & $1 ; 3 ; 4 ; \mathrm{G} ; \mathrm{T}$ & 0.0 & 0.0 & 0.0 & 0.0 & $0.0(0.0)$ & $2.5(0.3)$ \\
\hline isotig21033 & HpRDR6 & Regulation of megasporogenesis & $3 ; 4$ & 2.5 & 1.4 & 1.1 & 5.5 & $3.9(4.5)$ & $2.1(0.0)$ \\
\hline isotig18046 & HpSGS3 & Regulation of megagametogenesis & $3 ; 4$ & 8.5 & 7.1 & 6.7 & 8.1 & $5.3(0.7)$ & $6.4(5.5)$ \\
\hline isotig27635 & HpATREC8 & Cell specification and plant meiotic genes & $1 ; 6 ; \top$ & 0.5 & 0.0 & 3.1 & 3.9 & $0.0(0.0)$ & $1.9(1.5)$ \\
\hline isotig06846 & HpDMC1.1 & Plant meiotic genes & $6 ; T$ & 1.0 & 0.0 & 2.6 & 3.4 & $1.7(1.3)$ & $3.5(1.0)$ \\
\hline isotig06845 & HpDMC1.2 & Plant meiotic genes & $6 ; T$ & 1.8 & 0.0 & 3.0 & 4.1 & $1.9(1.7)$ & $4.1(1.5)$ \\
\hline isotig30894 & HpMND1 & Plant meiotic genes & $6 ; G ; T$ & 3.1 & 0.0 & 0.0 & 1.2 & $6.9(6.4)$ & $1.1(0.1)$ \\
\hline isotig31045 & HpMS5 & Plant meiotic genes & $6 ; T$ & 2.5 & 0.0 & 2.1 & 0.0 & $9.3(10.6)$ & $0.0(0.0)$ \\
\hline isotig32956 & HpAGP18 & Female gametogenesis, fertilization, seed development & 5 & 2.5 & 0.0 & 0.0 & 0.0 & $8.1(9.3)$ & $1.4(1.9)$ \\
\hline otig10679 & HpAGO5.1 & Regulation of megagametogenesis & 7 & 3.7 & 0.6 & 6.1 & 2.0 & 4.6( & $6.6(3.4)$ \\
\hline isotig15447 & HpAGO5.2 & Regulation of megagametogenesis & 7 & 5.4 & 13.0 & 6.6 & 8.2 & $4.4(1.0)$ & $4.6(3.5)$ \\
\hline isotig24484 & HpMET1 & Regulation of megagametogenesis and seed development & $3 ; 4 ; 8$ & 0.7 & 0.6 & 0.8 & 2.6 & $3.1(1.7)$ & $2.0(1.1)$ \\
\hline isotig09834 & HpDME.1 & Imprinted genes and imprinting related & $3 ; 4$ & 0.8 & 0.0 & 5.3 & 0.4 & $0.0(0.0)$ & $3.9(2.7)$ \\
\hline isotig09835 & HpDME.2 & Imprinted genes and imprinting related & $3 ; 4$ & 0.9 & 0.0 & 6.3 & 0.4 & $0.0(0.0)$ & $4.6(3.3)$ \\
\hline isotig31020 & НрMYB98 & $\begin{array}{l}\text { Regulation of megagametogenesis, fertilization, } \\
\text { seed development }\end{array}$ & $3 ; 4 ; 5 ; \top$ & 0.0 & 5.8 & 0.0 & 0.0 & $0.6(0.8)$ & $0.6(0.8)$ \\
\hline isotig23549 & HpDVPE & Female gametogenesis, fertilization, seed development & 5 & 0.0 & 0.0 & 2.1 & 0.0 & $2.6(0.5)$ & $3.9(1.2)$ \\
\hline isotig33211 & HpFUS2 & Female gametogenesis, fertilization, seed development & 5 & 0.0 & 0.0 & 1.0 & 0.0 & $0.0(0.0)$ & $6.1(8.6)$ \\
\hline isotig25989 & HplLA & Female gametogenesis, fertilization, seed development & 5 & 11.2 & 9.4 & 7.5 & 3.7 & $6.9(1.2)$ & $5.5(6.8)$ \\
\hline isotig23192 & HpAGL32 & Female gametogenesis, fertilization, seed development & 5 & 14.3 & 0.0 & 0.0 & 0.0 & $7.9(1.9)$ & $6.4(2.1)$ \\
\hline isotig11347 & HpSERK1 & Gametogenesis & $\mathrm{T}$ & 5.1 & 0.4 & 1.9 & 2.1 & $5.8(1.0)$ & $2.7(0.2)$ \\
\hline isotig11348 & HpSERK2 & Gametogenesis & $\mathrm{T}$ & 2.9 & 2.5 & 1.8 & 5.0 & $5.0(2.3)$ & $3.2(0.2)$ \\
\hline isotig32841 & HplAA32 & Maternal effect embryo arrest and gametogenesis & $\mathrm{G} ; \mathrm{T}$ & 0.8 & 0.0 & 0.0 & 0.0 & $4.8(0.3)$ & $0.0(0.0)$ \\
\hline isotig05393 & HpARF2 & Seed development and size regulation & 8 & 2.3 & 2.3 & 4.1 & 3.0 & $3.9(0.4)$ & $7.5(0.0)$ \\
\hline isotig29668 & HpAGL62 & Seed development and size regulation & 8 & 0.0 & 0.0 & 0.6 & 0.5 & $2.2(1.7)$ & $1.0(0.1)$ \\
\hline isotig29604 & HPTANMEI & Embryo defective phenotype and embryogenesis & $\mathrm{G} ; \mathrm{T}$ & 2.7 & 0.0 & 0.0 & 0.5 & $1.4(0.6)$ & $0.0(0.0)$ \\
\hline isotig25721 & HрAHP4 & Seed development and size regulation & 8 & 0.0 & 2.1 & 0.0 & 0.0 & $8.0(1.3)$ & $18.6(13.1$ \\
\hline
\end{tabular}

Genes were selected on the basis of their annotation. The number of reads, expressed as RPK (number of reads per kilobase of the reference CDS, normalized over the total number of sequences produced in the specified library and expressed as thousands of sequences), is displayed for each flower parts and for the two reproductive strategies (DB sources: 1, [12]; 2, [11]; 3 [5]; 4 [3]; 5, [4]; 6, [10]; 7, [13]; 8 [8]; G, GenBank; T, TAIR). Unigenes were deposited as TSA project under the accession GBXG00000000.

is implicated in the development and maintenance of MMC identity in the Arabidopsis ovule, was downregulated at early stages and up-regulated at late stages of pistil development in apomictic genotypes compared to sexual ones (Figure 6, panel A). Some genes potentially involved in ovule and cell identity, like $H p I N O$, did not reveal any differential expression between sexual and apomictic genotypes. Other genes potentially involved in cell identity, including $H p A N T$ and $H p B E L 1$, were down-regulated at early and late stages of pistil development in apomictic genotypes (Figure 6, panel A). The expression data of HpDMC1.2, HpMND and HpMS5 did show that these three genes are down-regulated in apomictic genotypes at early and late stages of pistil development (Figure 6, panel B). While HpDMC1.2 was differentially expressed between sexual and apomictic 


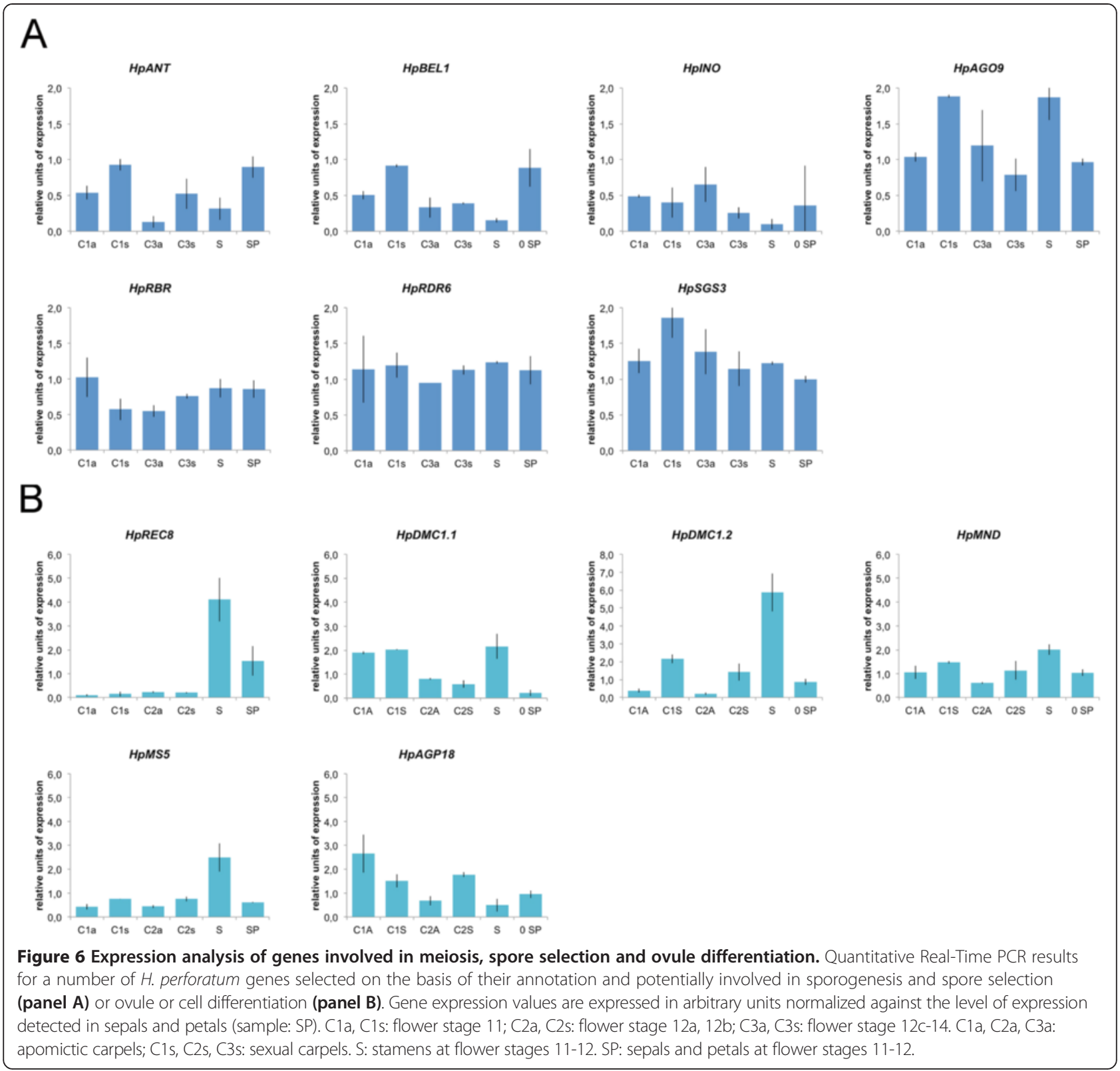

pistils, the related $H p D M C 1.1$ was expressed at comparable levels in apomict and sexual pistils (Figure 6, panel B).

Two Hypericum ARGONAUTE homologs of AtAGO5, both involved in megaspore selection and embryo sac development in Arabidopsis, showed no significant differences in expression levels between apomictic and sexual pistils (Figure 7, panel A). Concerning genes that in Arabidopsis control DNA methylation in sporophytic and gamethophytic tissues, HpDME1 was found upregulated in apomictic genotypes at early and late stages of pistil development, and to a lower extent also $H p D M E 2$ was found up-regulated in apomictic genotypes (Figure 7, panel A). In addition, HpMET1 proved to be down-regulated in apomictic genotypes compared to sexual ones at both stages of pistil development (Figure 7, panel A). The expression of several genes that are involved in embryo sac and embryo, and endosperm development (Table 6) was also tested in the same set of pistils from sexual and apomictic genotypes (Figure 7, panel B). It is worth mentioning that both HpSERK1 and $H P S E R K 2$ were differentially expressed in genotypes with antagonist reproductive features, being down-regulated at early and late stages of pistil development in apomictic genotypes (Figure 7, panel B). Moreover, with the only exception of HpdVPE and HpILA, whose expression in pistils appeared to differ in apomictic and sexual genotypes at late developmental stages, most of the tested genes did not reveal expression changes (Figure 7, 


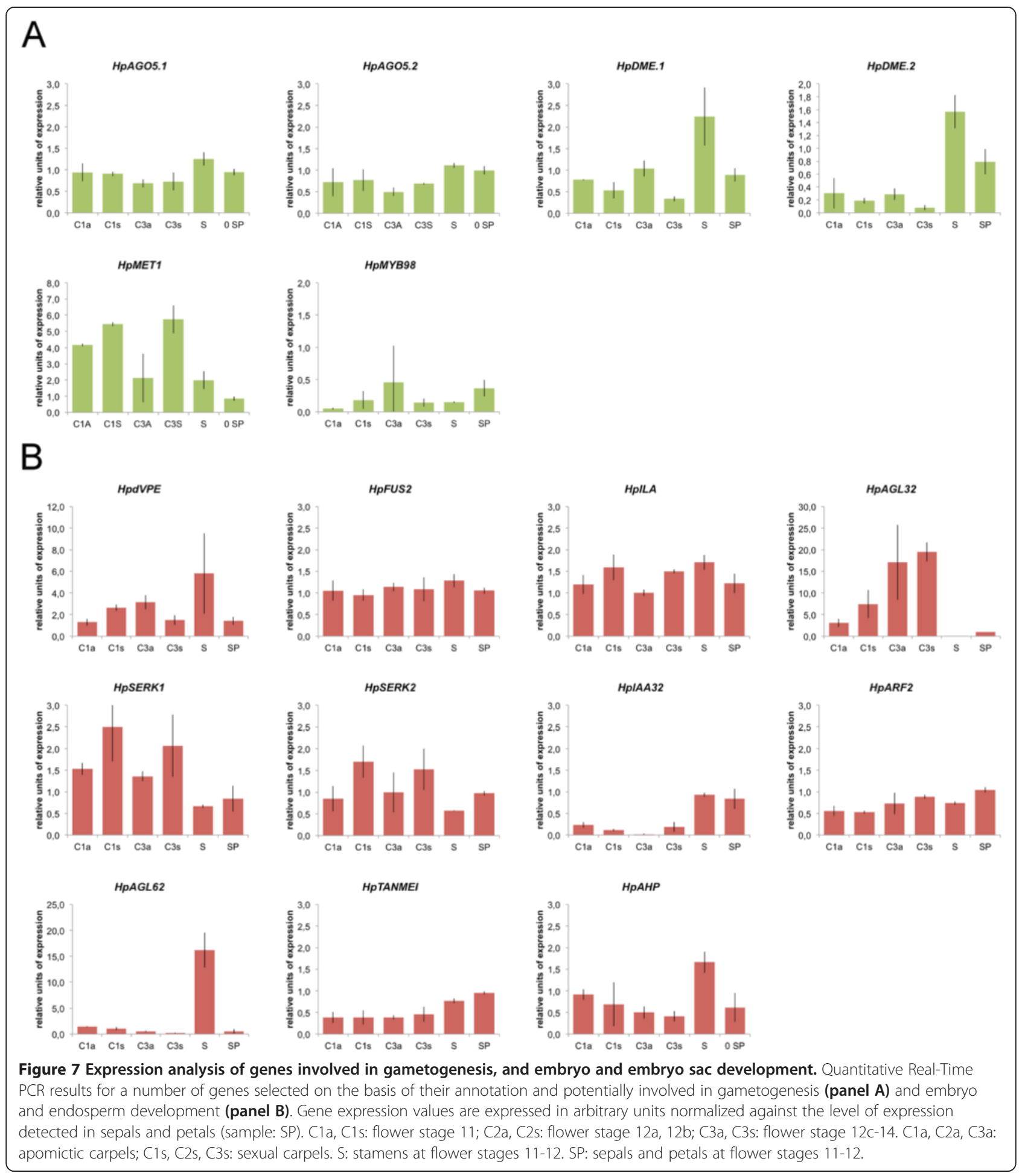

panel B). The two genes HpMYB98 and HpAGL32, known in Arabidopsis to be specifically expressed in ovule domains including the embryo sac and the endothelium, were found similarly expressed in apomictic and sexual pistils (Figure 7, panels A and B, respectively).

\section{Computational investigation of flower and seed-related} genes based on Arabidopsis microarray-based reference transcriptome

The possibility to discover additional genes involved in molecular pathways leading flower and seed development 
was addressed by comparative approaches focused on $A$. thaliana flower and seed transcriptomes as references (see Additional file 1: Table S7). To do so we created an Arabidopsis microarray-based reference transcriptome, based on the expression data of 20 published microarray experiments performed in $A$. thaliana by using flower tissues at developmental stages preceding pollination (e.g., whole flowers, carpels and ovules, anthers and pollen grains). In addition to these, the microarray-based reference transcriptome was enriched with the data of 17 publically available microarray experiments performed on A. thaliana pollinated flowers and seeds (e.g., whole seeds, endosperm, embryo) (Additional file 1: Table S7).

Based on the published data of the A. thaliana microarrays, our reference dataset contained 20,372 genes expressed in at least one of the microarray experiments considered in our study, irrespectively of their expression values (Table 7). This reference dataset was used to set the architecture of pollinated and non-pollinated flowers in MapMan. On the other side, based on our BLASTX results, we could use approximately $60 \%$ and $63 \%$ of genes expressed in the A. thaliana flower and seed (12,035 and 12,003 hits, respectively) as they produced significant matches with one or multiple Hypericum unigenes (Table 7).

We found that 191 Hypericum transcripts were expressed only before pollination in the Arabidopsis flower (see Additional file 3: Figure S2). Using the same approach, we could detect 65 Hypericum unigenes, which appeared to be expressed upon fertilization in Arabidopsis flowers (Additional file 3: Figure S2, panel A). Interestingly, a number of Hypericum unigenes were shown to be specific to certain seed structures in Arabidopsis (Additional file 3: Figure S2, panel B). This was the case of HpAGL80/FEM111 (AT5G48670) and HpEMB2271 (AT4G21130), whose Arabidopsis putative orthologs appeared to be expressed in the Arabidopsis endosperm.

An overview of metabolic and regulative pathways active during flower development was defined by mapping the Hypericum flower unigenes to the metabolic and regulative environments defined by MapMan (see Additional file 4: Figure S3). Particular emphasis was given to the maps: cell regulation overview and cellular response pathways (Additional file 4: Figure S3). A total of 74 apomictic-specific and 137 sexual-specific gene transcripts, whose expression was restricted to Hypericum flowers, had homologues in the Arabidopsis flower transcriptome (see Additional file 4: Figure S3, indicated as light-blue and green squares, respectively). Sixty-five Hypericum unigenes matched Arabidopsis seed-specific genes (see Additional file 4: Figure S3, red squares) whose annotations were related to the following categories: transcription factors (21 hits; $30 \%$ ), protein modification (5 hits; 7\%) and degradation (10 hits; 14\%), receptor kinases ( 9 hits; 13\%), as well as the synthesis and metabolism of hormones (13 hits; 19\%; Additional file 4: Figure S3). Among the genes related to seed development in Arabidopsis, the homolog of the B3 family transcription factor AT1G26680 was uniquely present in the sexual libraries in H. perforatum (see Additional file 4: Figure S3, orange squares). Similarly, the expression of the homolog of the embryo-specific zinc-finger transcription factor AT5G0750 (TZF6), which is required for heart-stage embryo formation in the Arabidopsis seed, was present in the apomictic, but not in sexual carpels (see Additional file 4: Figure S3, violet squares).

\section{Discussion}

\section{De novo sequencing and assembly of the Hypericum flower transcriptome}

$H$. perforatum L. is a medicinal plant with reproductive behavior ranging from highly or nearly obligate apomixis (frequent) to complete sexuality (rare) in natural populations [19,31]. Even though $H$. perforatum is not a traditional model system for plant biology studies, it is a suitable system for the study of apomeiosis and parthenogenesis $([17-19,26])$. To do so, we approached the flower transcriptome with two sequencing efforts conceived for

Table 7 Coverage estimates of the Hypericum flower transcriptome by comparative analysis with Arabidopsis data

\begin{tabular}{lllll}
\hline Plant organ (Arabidopsis) & $\begin{array}{l}\text { Experiments } \\
\text { (replicates) }\end{array}$ & $\begin{array}{l}\text { Database size } \\
\text { (\# sequences) }\end{array}$ & Hits (\%) & $\begin{array}{l}\text { Hits with array elements } \\
\text { that are not expressed in } \\
\text { the specific experiments (\%) }\end{array}$ \\
\hline Flower - not pollinated & $21(54)$ & 20,032 & $11,970(59.8)$ & $331(2.7)$ \\
Flower - pollinated & $16(40)$ & 19,406 & $11,844(61.0)$ & $457(3.7)$ \\
Flower - overall & $37(94)$ & 20,372 & $12,035(59.1)$ & $266(2.2)$ \\
Seed & $50(98)$ & 20,679 & $12,003(58.0)$ & $298(2.4)$ \\
Embryo & $6(12)$ & 17,275 & $10,995(63.6)$ & $1,306(10.6)$ \\
Endosperm & $16(34)$ & 19,247 & $11,451(59.5)$ & $850(6.9)$ \\
\hline
\end{tabular}

The transcriptome coverage was estimated by comparing annotated Hypericum sequences with publically available Arabidopsis microarray data (http://www.ebi.ac. uk/arrayexpress/). For each plant component, such as flower and seed, the number of microarray experiments and replicates that were used to estimate the size of each database are reported. The recovered hits indicate the number (and percentage) of database sequences that matched Hypericum accessions. For each plant component, the number of array elements that are not expressed in the specific experiments (non-significant hybridization signals), but that produced significant matches with Hypericum sequences is also reported. 
better understanding how and where differential gene expression occurs between both reproductive forms. Hence, we sequenced cDNA collected from single flower organs (i.e., sepals and petals, stamens and carpels) at different stages of flower development, and linked these data to RNAseq data from whole flowers collected from two different obligately-sexual genotypes and two different highly-apomictic genotypes. These efforts led to the assembly of 1.47 million sequence reads to produce 60,428 unigenes, which proved to be a valuable tool for the study of the Hypericum flower transcriptome. Starting from these data, we were able to annotate and characterize 36,988 transcripts found expressed in male and/or female reproductive organs, including tissues or cells of sexual and apomictic flower buds. Our approach of sequencing flowers from two fully obligate sexual genotypes and two unrelated highly apomictic genotypes, in addition to different flower parts dissected from a facultativelyapomictic accession, enabled us to approach the complexity of the flower transcriptome according to its main reproductive organs as well as for alternative reproductive behaviors.

The assembly of such a large number of unigenes provided the possibility of testing for the presence of alternative alleles or splicing variants that could have been sequenced and assembled independently. To verify this thesis we investigated the unigenes aligning to the genomic region comprised in the BAC sequence HM061166.1 and surrounding the HAPPY locus [18]. Nine genes included in the BAC clone aligned to multiple unigenes sequenced from the flower libraries. Despite being sufficiently dissimilar to escape the assembly procedure, the fact that multiple unigenes aligned to the same region could indicate the sequencing of alternative alleles or splicing variants. This could be the case of the BAC regions encoding for the gene products WRKY, DGCR and ARI7, as well as EXP1, RimL and PG. Findings of nucleotide substitutions concentrated in the 3'end of the alignments in at least $50 \%$ of investigated regions could be an additional clue that analyzed SNPs are robust and not attributable to sequencing artifacts.

On average, the nucleotide diversity of multiple unigenes mapping to the genomic window surrounding the HAPPY locus [18] was comparable to that estimated for the ITS regions used as external reference [36]. Despite the limitation due to the absence of mapping and quantitative expression data, the finding of nucleotide diversity shared by unigenes and genomic reference stretches comparable to the extent showed by ITS regions suggests that most of the unigenes may resemble allele variants rather than long-lasting duplicated genes (which would have been associated with higher nucleotide diversity, assuming enough time for independent accumulation of mutations). We are aware that coverage provided by the technology used in the study is lower than that recommended for robust variant detection analysis [35], and hence our estimates of nucleotide diversity could be affected by the presence of undetected of sequencing errors. Nevertheless, if we consider that the error rate is dependent on the sequencing method, its distribution across all loci of the BAC clone would be substantially uniform. Hence, we believe that our finding that unigenes aligning on ARI7-like displayed a nucleotide diversity largely exceeding that calculated on the surrounding regions of the BAC clone (0.013) is worth mentioning. This finding is interesting as we consider that ARI7 contains the actual marker co-segregating with apospory in $H$. perforatum and is among those few genes predicted from HM061166.1 (Additional file 1: Table S3).

Finally, taking into account the low genetic diversity existing within unigenes and CDS predicted from the BAC clone HM061166.1, the finding of alignment variants displaying the insertion or deletion of gene sequence traits in the unigenes provides a first indication for the presence of splice variants in sequences encoded by genes surrounding the HAPPY locus (Figure 2, see also Additional file 4: Figure S3). The allelic nature of these unigenes will be eventually confirmed by the availability of mapping data.

\section{Genes related to plant reproductive organs and seed formation}

De novo sequencing of the $H$. perforatum flower transcriptome was attempted to identify genes related to plant reproduction, including transcripts specifically or preferentially expressed in anthers and/or pistils, and possibly differentially expressed between sexual and asexual meiosis and gametogenesis. Computational in silico annotation of flower transcripts provided us with an insight into the molecular mechanisms of various biological processes, including meiosis and gametogenesis.

Particular attention was paid to the annotation of genes important for cell specification $[3,12]$ and genes whose mutants affect gametophyte development $[5,8,11]$. The orthologs of genes whose products are possibly involved in restricting cell-fate in ovules or gametophytes were detected (Table 6; Figure 6). Genes that are involved in regulation of the correct number of cell divisions occurring during megagametogenesis (RBR) [37] or the correct positioning of nuclei within the embryo sac (BLH1/eostre) [38] were also detected in our datasets. Accordingly, transcripts whose expression are thought to be crucial for selection of the functional megaspore and its cell identity (AGP18) [39], or those involved in proper specification of gametes and cell fates, including LIS [7], CLO [40] and ATO [40], were sequenced and annotated. 
Overall, these findings demonstrated that our approach could successfully identify gene products whose expression is associated with ovule and seed development, and restricted to a few number of cells [41,42]. For example, we were able to identify the homolog of MYB98 (Table 6; Figure 7), which in Arabidopsis is expected predominantly in the synergid cells and is considered to be critical for synergid differentiation in this species [41]. It is remarkable that $H p M Y B 98$, a gene associated with proper differentiation of synergid cells [3-5,17], was characterized by high levels of expression variation (compared to sexuals) in the pistils collected at late developmental stages from apomictic accessions (Table 6; Figure 7). As the sexuals and apomicts compared here were of identical ploidy, elevated gene expression variation in apomicts could be consistent with a number of factors including genetic background differences between genotypes [29], trans-acting regulatory variation in the hybrids or genomic differences having arisen due to sexuality versus apomixis [43]. Assuming that PCR efficiency was comparable in the different tissues, the high level of variation recorded in apomictic pistils at stages of late gametogenesis could be the result of spatial or temporal mis-expression of some components of the genetic pathways acting during gametogenesis.

The orthologs of genes that were previously associated with seed and endosperm abortion and anomalies in zygote development, including IAA32/MEE10, dVPE, ILITHYIA, SERK1 and SERK2 among others [4,44], were sequenced and studied in greater details by RT-qPCR. Quantitative expression studies demonstrated that apomictic and sexual pistils have different relative abundance of transcripts encoded by these genes (Table 6; Figure 7). The finding that multiple genes that are involved in seed and endosperm development are differentially expressed between sexual and apomictic pistils implies that entire reproductive gene pathways are modulated in their timing and levels during apomictic development.

Validation using RT-qPCR assays confirmed that HpMET1 [45] and HpDME [46] are differentially expressed among the different flower parts (Figure 7) while only HpMET1 appeared to be differentially expressed in apomictic and sexual pistils at developmental stages corresponding to gametogenesis. In Arabidopsis MET1 absence leads to removal of the silencing methylation marks and the expression of genes (e.g., FIS2 and FWA) involved in the regulation of endosperm development [5]. Accordingly, [45] reported that MET1 is implicated in the repression of endosperm development in the absence of fertilization in Arabidopsis [45], while [47] provided data in support of the idea that a DNA methylation pathway in maize likely plays a critical role in the differentiation between apomictic and sexual reproduction.
Hence, the reduction in the expression of HPMET1 in apomictic pistils further support that variations of DNA methylation marks might be linked to gametophyte development in apomictic $H$. perforatum.

\section{Identification and expression analysis of candidate genes for apomixis}

Apomixis, a form of asexual seed production is characterized by i) meiotically-unreduced egg cell formation (apomeiosis); ii) development of an embryo without the fertilization of the egg cell (parthenogenesis); and iii) endosperm development with (pseudogamous endosperm) or without (autonomous endosperm) fertilization of the central cell [48].

The most abundant GO terms found associated with the unigenes differentially expressed between sexual and apomictic accessions were those associated with transport, response to stress, biotic and abiotic stimuli, and DNA metabolic process and DNA binding (Figure 4). The number of enriched GO terms specifically related to plant reproduction (i.e., meiosis, gametogenesis, and embryonic and post-embryonic development) was relatively low in either sexual or apomictic genotypes (Figure 4). Nevertheless, our findings that unigenes found only in sexual or apomictic libraries were annotated as cell cycle, cell growth, cell differentiation, as well as embryonic and post-embryonic development (Figure 4) could provide some additional indications supporting the hypothesis that apomixis relies upon spatial or temporal mis-expression of genes acting during female sexual reproduction [27,30,49-52]. Hence a better understanding of the genetic mechanisms underpinning the switch from sexual to apomictic reproductive strategies could be greatly facilitated by the study of genes primarily involved in the normal progression of sporogenesis, gametogenesis and embryo development. In light of this, we examined whether apomictic seed development was characterized by expression by genes known to be important for the regular progression of gametogenesis and seed development in sexual plants.

One critical finding was the observation that gene products involved in cell differentiation and cell growth, as well as embryonic and post-embryonic development were overrepresented in the two sexual libraries (Figure 4). A closer expression analysis of multiple genes involved in meiosis (HpDMC1, HpMS5, HpMND) or megaspore selection (HpAGP18) indicates a relative decrease in mRNA abundance in apomictic pistils (Figure 6). The analysis of two distinct $H p D M C 1$-like unigenes indicated that their respective mRNAs had contrasting expression patterns, and while $H p D M C 1 b$ shows clear differential expression in all considered developmental stages of the pistil, the expression of $\mathrm{HpDMC1a}$ is similarly expressed in both apomictic and sexual pistils (Figure 6). Since cytological and 
cytometric data $[19,29]$ indicate that apomictic plants in $H$. perforatum are characterized by a low occurrence of reduced embryo sacs, the decreased expression of critical genes for normal progression of meiosis supports the hypothesis that apospory in this species could be associated to a low rate of successful meiosis.

It has been hypothesized that the molecular switch from sex to apomixis is associated with gene-specific silencing mechanisms associated with chromatin remodeling factors or trans-acting and heterochromatic interfering RNAs involved in both transcriptional and post-transcriptional gene regulation [27]. Recently, a specific route for cell specification and embryo sac development was proposed to occur through the action of different ARGONAUTE (AGO9 and AGO5) genes in Arabidopsis [13,14] and maize [15]. Similarly, mutations in RDR6 or SGS3, two genes involved in the small RNA pathway, are known to result in a relaxation of gametic cell identity and fate in the ovule, leading to changes in cell fate of somatic initials in the nucellar tissue, which differentiate into gametic cells without undergoing meiosis and which can furthermore initiate female gametogenesis through the activation of TEs [14]. The RTqPCR analyses performed here did not demonstrate differences in the abundance of RDR6 and SGS3 in sexual and apomictic pistils collected at developmental stages spanning female meiosis and gametogenesis (Figure 6). Similarly, no significant differences were detected in the expression of $\mathrm{HpAGO5}$ in the same plant tissues (Figure 7). Alternatively, since RT-qPCR analyses were performed on whole pistils, we cannot exclude the possibility that small expression differences consistent with gene expression in specific cell types of the ovule might have been lost. This

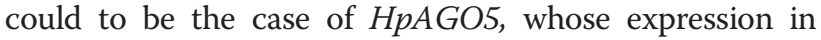
Arabidopsis is expected in the nucellus, where it activates a non-cell autonomous mechanism by promoting the initiation of gametogenesis in the functional megaspore.

Our RT-qPCR data indicated that mRNA levels of HpAGO9 are significantly decreased in apomictic pistils at the earlier developmental stages (Figure 6). Reduced expression of $H p A G O 9$ in pistils retaining a high level of apospory is noteworthy since $A G O 9$ is involved in restricting the acquisition of gametophyte cell fate in the sub-epidermal layer of the ovule nucellus in Arabidopsis [14], and the phenotype documented for ago9 Arabidopsis plants is remarkably similar to the aposporic gametophyte development [14].

Such considerations of the expression and activity of sRNAs and non-coding RNAs would support the idea that the coding fraction of the transcriptome could be only one side of the coin, and that small and non-coding RNA sequences could represent a meaningful component yet to be fully understood in the frame of gametes and seeds development.

\section{Conclusions}

The availability of NGS technologies is raising the ease at which complex phenotypes and processes in nonmodel organisms can be analyzed as a consequence of the increased of throughput and availability of reference DNA and RNA datasets. In our study we were able to annotate and characterize 36,988 transcripts found expressed in male and/or female reproductive organs, including tissues or cells of sexual and apomictic flower buds. Our approach of sequencing whole flowers at different developmental stages from two fully obligate sexual genotypes and two unrelated highly apomictic genotypes, in addition to different flower parts dissected from a facultatively apomictic accession, enabled us to analyze the complexity of the flower transcriptome according to its main reproductive organs (i.e., sepals, petals, stamens and carpels) as well as for alternative reproductive behaviors (i.e., sexual $v s$. apomictic).

Our findings that unigenes found only in sexual or apomictic libraries were annotated as cell cycle, cell growth, cell differentiation, as well as embryonic and post-embryonic development provided additional indications supporting the hypothesis that apomixis relies upon spatial or temporal mis-expression of genes acting during female sexual reproduction. This hypothesis is further strengthened by our RT-qPCR data, demonstrating that genes expressed in sporophytic and gametophytic cell types and primarily involved in the normal progression of sporogenesis, gametogenesis and embryo development are differentially expressed between apomictic and sexual pistils.

Overall, the results collected in this research represent a critical step toward a better understanding of the genetic and molecular mechanisms leading to gametophyte and gamete determination and development in our model species, in either sexual or apomictic plant genotypes. Data presented here pave the way for whole transcriptome studies aimed at the identification and definition of transcriptome changes associated with the development of male and female reproductive organs by giving particular emphasis to meiosis, the formation of gametes and, more generally, to the processes associated with plant reproduction and seed formation in $H$. perforatum.

\section{Methods}

Plant materials

Hypericum perforatum L. plants from two naturally occurring tetraploids $(2 n=4 \times=32)$ and three induced tetraploids $(2 n=4 \times=32)$ were used for the sequencing approaches (Table 1). For the production of induced tetraploids, plants of the diploid sexual line $\mathrm{R} 1$, reselected from the tetraploid apomictic cultivar 'Topaz', were converted to auto-polyploids by colchicine application, as 
reported by Schallau et al. [18]. Briefly, seeds were imbibed in water for $24 \mathrm{~h}$, placed on filter paper soaked with an aqueous solution of $0.2 \%$ colchicine for $24 \mathrm{~h}$ and then planted in soil for germination. The $\mathrm{C} 0$ plants that survived this treatment were self-pollinated and their progenies $(\mathrm{C} 1)$ were screened for tetraploid plants. Sexual tetraploid $\mathrm{C} 1$ and $\mathrm{C} 2$ plants were used for further crosses with tetraploid apomictic pollinators and their progenies were then screened for the reproductive phenotype $[18,29]$. The reproductive mode of all $H$. perforatum accessions was estimated by flow cytometric screening of 48 single seeds as indicted by Matzk et al. [31].

We constructed a total of 8 different cDNA libraries. A single cDNA library was obtained from whole flowers collected across developmental stages 1-12 for each of the following accessions: HP13EU and HP36EU (sexual plant accessions), HP39EU and HP1973US (apomictic plant accessions). These four cDNA libraries were sequenced twice. An organ-specific cDNA library was also produced from the facultative apomictic accession HP4/ 13 for each of the following flower parts: buds (whole buds, length $<3.0 \mathrm{~mm}$, corresponding to Arabidopsis flower stages 1-10), carpels (bud length $>3.0 \mathrm{~mm}$, corresponding to Arabidopsis flower developmental stages 11-12), stamens (bud length $>3.0 \mathrm{~mm}$, corresponding to Arabidopsis flower developmental stages 11-12) and sepals/petals (bud length $>3.0 \mathrm{~mm}$, corresponding to Arabidopsis flower developmental stages 11-12) [18,53]. These four cDNA libraries were sequenced once.

\section{cDNA synthesis, normalization and pyrosequencing}

Total RNA was extracted from whole flowers and flower parts by using the Spectrum ${ }^{\mathrm{Tm}}$ Plant Total RNA Kit (Sigma-Aldrich) following the protocol provided by the manufacturer. The eventual contamination of genomic DNA was avoided by the optional DNase I (Sigma-Aldrich) treatment. Poly $(\mathrm{A})+$ mRNA was isolated from total RNA using the Ambion MicroPoly(A) Purist Kit according to the manufacturer's instructions (Life Technologies, Darmstadt, Germany) and the integrity and quantity was verified using an Agilent 2100 Bioanalyser and RNA Nanochips (Agilent Technologies, Santa Clara, USA). In order to avoid over representation of the most commonly transcribed genes and to maximize sequence diversity, normalized, full-length enriched cDNA libraries were generated using a combination of the SMART [54] cDNA library construction kit (Clontech, Takara, Saint-Germainen-Laye, France) and the DSN normalization method [55] implemented in the Trimmer Direct cDNA normalization kit (Evrogen, BioCat, Heidelberg, Germany). The procedure generally followed the manufacturer's protocol but included several important modifications, essentially as previously described in more detail [56]. Optimization of the complete cDNA normalization procedure, such as the number of thermocycles for ds-cDNA synthesis, was essentially carried out as described by Vogel and Wheat [57]. Each of the resulting ds-cDNA pools was purified and concentrated using the DNA Clean and Concentrator kit (Zymogen) and size fractionated with SizeSep 400 spun columns (GE Healthcare) that resulted in a cutoff at $\sim 200$ bp. To test for normalization efficiency, a fraction of the full-length-enriched cDNAs were cut with SfiI and ligated to pDNR-Lib plasmid (Clontech). Ligations were transformed into E. coli ELECTROMAX DH5 $\alpha$-E electrocompetent cells (Invitrogen). For each cDNA library, appr. 200 bacterial colonies were grown in 96 deep-well plates, plasmid mini-preparations were performed using the 96well robot plasmid isolation kit (NextTec) on a Tecan Evo Freedom 150 robotic platform (Tecan) and Singlepass sequencing of the 5 ' termini of cDNA libraries was carried out on an ABI $3730 \mathrm{xl}$ automatic DNA sequencer (PE Applied Biosystems). Vector clipping, quality trimming and sequence assembly using stringent conditions (e.g., high quality sequence trimming parameters, $96 \%$ sequence identity cutoff, 35bp overlap) was done with the Lasergene software package (DNAStar Inc.). For direct sequencing, approximately $1 \mu \mathrm{g}$ of each SfiI-digested normalized cDNA sample was sheared via nebulization into small fragments. The eight shotgun cDNA libraries were grouped in two plates and sequenced on the Roche 454 FLX platform using Titanium chemistry (Roche Diagnostics Corporation, Basel, Switzerland).

\section{Bioinformatics - EST processing protocol}

All high quality reads generated from the two sequencing reactions were assembled in a single reference transcriptome. The assembly was done with gsAssembler (Newbler v2.7, Roche Diagnostics Corporation, Basel, Switzerland) by considering the default parameters, including a minimum read length: 20 ; seed length: 16 ; seed step: 12; minimum overlap length: 40 and minimum overlap identity: 90 . Both contigs and singletons originated from this procedure were then used as input files in a second assembly step in which all parameters were maintained equal to the previous assembly, except that the minimum overlap length was lowered to $20 \mathrm{bp}$.

For each single library and assembled sequence, the relative abundance of predicted unigenes was calculated by counting the number of raw reads taking part in the assembly. A unigene was considered expressed in one library if at least one sequence derived form the specified library was included in the assembly of the considered unigene. Transcriptome overlaps and compositions were displayed with Venn diagrams using the web tool venny (bioinfogp.cnb.csic.es/tools/venny/index.html; [58]).

A BLASTX-based approach was used to compare the Hypericum sequences to the $\mathrm{nr}$ database and to annotate the assembled Hypericum unigenes, using the BLAST 
v2.2.29+ downloaded from NCBI, National Center for Biotechnology Information (http://www.ncbi.nlm.nih.gov/).

The $H$. perforatum clone BAC $25 \mathrm{H} 09$ genomic sequence containing the apospory-linked marker and covering the apospory-associated region (GenBank accession number HM061166.1) was previously identified and annotated for several predicted genes, including a variety of transcription factors and retrotransposons [18]. Alignments of unigenes to the BAC sequence HM061166.1 were performed with software the CLC Genomics Workbench v7 (QIAGEN). Statistics on genetic diversity and distribution of the average number of nucleotide substitutions per site (Dxy) were performed with the software DNAsp v5.10.1 [59]. Values of Dxy were extracted from the package of statistics: DNA divergence between populations (sliding window: $100 \mathrm{bp}$; overlaps: $25 \mathrm{bp}$ ).

To annotate all assembled unigenes (contigs and singletons), a BLASTX-based approach was used to compare the Hypericum sequences to the $\mathrm{nr}$ database downloaded from the NCBI (http://www.ncbi.nlm.nih.gov/). Moreover, the GI identifiers of the best BLASTX hits, having E-value $\leq 1$ E-09 and similarity $\geq 70 \%$, were mapped to UniprotKB protein database (http://www.uniprot.org/) in order to extract Gene Ontology (GO, http://www. geneontology.org/) and KEGG orthology (KO, http:// www.genome.jp/kegg/) terms for further functional annotations. The BLAST2GO software v1.3.3 (http://www. BLAST2go.com; [60]) was used to reduce the dataset to the GOslim level (goslim_plant.obo) and perform basic statistics on ontological annotations, as reported by [61].

\section{Plant reproduction database}

The plant reproduction database was generated based on the refseq protein sequence repository at GenBank that could be downloaded by browsing the dataset with opportune key words such as ("embryo defective"[All Fields], "maternal effect embryo arrest"[All Fields], "unfertilized embryo sac"[All Fields], "gametophytic factor"[All Fields]). Searches were restricted to the Plants by using the option "Viridiplantae"[Organism]. In a parallel approach, all sequences annotated as "meiosis", "gametophyte development", and "embryogenesis" were downloaded from TAIR (http://www.arabidopsis.org/). Finally, a number of protein sequences whose gene products were studied and linked to "cell specification", "meiosis", "gametogenesis" and "seed development" [3-5,10-12] were downloaded from Genbank and added to the previous set. On the whole, the plant reproduction database considered 811 non-redundant Arabidopsis gene loci.

\section{Validation of sequencing data by quantitative Real-Time RT-PCRs}

Plant materials were selected according to the genetic and cyto-histological bases of apospory recently described for
Hypericum perforatum $[18,19]$. The reproductive mode of all $H$. perforatum accessions was estimated by flow cytometric screening of 48 single seeds as indicted by Matzk et al. [31]. Samples were collected in 3 biological replicates, from different plant accessions (Table 1). RNA extractions were conducted using the Invisorb Spin Plant RNA Mini Kit (Sigma-Aldrich). cDNA synthesis was conducted using the SuperScript ${ }^{\circ}$ VILO $^{\text {mo }}$ cDNA Synthesis Kit (life technologies), following the indication of the supplier. Primers used in the quatitative RT-PCR experiments are reported on Additional file 1: Table S8. Expression analyses were performed using the thermal cyclers StepOne and 7300 Real-Time PCR System (Applied Biosystem), equipped with 96- and 384-well plate systems, respectively, with the SYBR green PCR Master Mix reagent (Applied Biosystem). The amplification efficiency was calculated from raw data using the OneStep Analysis software (Life Technologies). Amplification performances expressed as fold change were calculated by the $\Delta \Delta \mathrm{Ct}$ method using HpTIP4 as housekeeping gene [62]. Error bars indicate the standard deviation observed among the three biological replicates.

\section{Computational investigation of flower and seed-related genes based on Arabidopsis microarray-based data}

An Arabidopsis microarray-based reference transcriptome was created using the Arabidopsis-expressed sequences, downloaded from publically available experiments. Microarray data sets were downloaded from the arrayexpress data repository at EBI (http://www.ebi.ac.uk/arrayexpress/ experiments/) and used to infer the most probable Arabidopsis transcriptome to be used as a reference (Additional file 1: Table S7). Probes were considered expressed when present in at least one experiment, considering a $\mathrm{p}$-value lower than 0.05 .

MapMan (http://mapman.gabipd.org/web/guest/mapman; [63]) analyses were performed by using the Hypericum dataset properly rearranged as input files. Briefly, the Arabidopsis proteome was downloaded from the TAIR, The Arabidopsis Information Resource database (ftp://ftp.arabidopsis.org/home/tair/Sequences/BLAST_ datasets/TAIR10_BLASTsets/) and set as reference database for local BLASTX analysis performed using the $H$. perforatum transcriptome dataset as the query. AGI codes relative to Arabidopsis putative homologous genes (E-value cut-off: 1.0E-9) were recovered by each tabular BLASTX result (BLAST-2.2.25+ argument: -outfmt 6) and used to download the corresponding ATH1121501 genechip identifiers (Affymetrix) from TAIR (http:// www.arabidopsis.org/tools/bulk/microarray/index.jsp) (for more details see [61]). MapMan analyses focused on maps: Regulation overview and Cellular response overview, which were used to graphically display the data. 


\section{Availability of supporting data}

Raw sequences files were made available for download from SRA with accession numbers: SRR1646951, SRR1646953, SRR1646955, SRR1646956, SRR1647632, SRR1647633, SRR1647673, SRR1647674, SRR1647677, SRR1647678, SRR1647713, SRR1647714. Sequences of unigenes investigated by Real-Time RT-qPCR along with unigenes aligned to the BAC sequence HM061166.1 were deposited as Transcriptome Shotgun Assembly project, which has been deposited at DDBJ/EMBL/GenBank under the accession GBXG00000000. The version described in this paper is the first version, GBXG01000000.

\section{Additional files}

Additional file 1: Table S1. Descriptive statistics of the sequenced Hypericum flower libraries. Table S2. Main statistics concerning the BLASTX sequence comparisons. Table S3. BLAST matches produced by comparing the assembled unigenes with the CDS deduced from the Hypericum BAC clone HM061166.1. Table S4. Abundance of unigene sequences matching the Hypericum BAC clone HM061166.1. Table S5. Ontological annotations ascribed to the Hypericum flower transcripts. Table S6. Hypericum unigenes that show high similarity with Arabidopsis genes annotated for terms related to plant reproduction sensu lato. Table S7. Publically available Arabidopsis microarray experiments used for comparisons with Hypericum flower transcriptomes. Table S8. Primers used in all Real-Time RT-PCR experiments (for additional information on each table, please see details reported in the additional file itself).

Additional file 2: Figure S1. Genetic diversity computed for the unigenes aligning to the Hypericum BAC clone HM06166. Alignments of multiple unigenes to the sequence extracted from HM61166.1 and the corresponding predicted CDS. Nine genes producing alignments with multiple unigenes are shown. For each alignment, the distribution of the average number of nucleotide substitutions per site (Dxy) calculated between unigenes and the aligned region of HM61166.1 is shown.

Additional file 3: Figure S2. Hypericum unigenes matching Arabidopsis flower and seed transcripts. Venn diagrams that graphically represent the distribution of unigenes matching an Arabidopsis gene model included on the Arabidopsis microarray-based reference transcriptome. A: main developmental stages (A: fertilized flowers, non fertilized flowers) considered in the Arabidopsis microarray-based reference transcriptome. B: main flower and seed organs included on the Arabidopsis microarray-based reference transcriptome.

Additional file 4: Figure S3. Overview of Hypericum transcripts involved in regulation and cellular response processes. Graphical representation of the Hypericum flower and seed transcriptomes produced with MapMan. Blue squares indicate significant matches with genes expressed in the Arabidopsis flower. Light blue: match within the flower, but found only in apomictic libraries; green: match within the flower, but found only in sexual libraries. Red squares indicate specific matches in the Arabidopsis seed dataset. Violet: seed library and found only in apomictic libraries; Orange: seed library and found only in sexual libraries. Black: sequences that could not find a match in the flower or seed reference databases.

\section{Abbreviations}

BAC: Bacterial artificial chromosome; BLAST: Basic local alignment search tool; CDS: Coding sequence; HAPPY: Hypericum APOSPORY; ITS: Internal transcribed spacers; NCBI: National center for biotechnology information; NGS: Next generation sequencing; GO: Gene ontology; RPK: Number of reads per kilobase; RT-qPCR: Reverse transcription quantitative polymerase chain reaction; SNP: Single nucleotide polymorphism; TAIR: The arabidopsis information resource.

\section{Competing interests}

The authors declare that they have no competing interests.

\section{Authors' contributions}

GG conceived and carried the computational and expression analysis, and prepared the first draft of the manuscript. HV carried out the CDNA synthesis and normalization. TFS conceived the study, participated to its design and coordination, and helped to accomplish the final form of the manuscript. GB conceived the study, participated to its design and coordination, and helped to accomplish the final form of the manuscript. All authors read and approved the final manuscript.

\section{Acknowledgements}

The authors want to thank COST Action FA0903 "Harnessing Plant Reproduction for Crop Improvement." This research was supported by the following grants: Research Project for Young Researchers of the University of Padova (year 2010), "Comparative and functional genomics for cloning and characterizing genes for apomixis" (code: GRIC101130/10), Principal investigator: Giulio Galla; Academic Research Project of the University of Padova (year 2012), "Transcriptomics of reproductive organs in model species for comparative analysis of the genetic-molecular factors characterizing sexual and apomictic processes" (code: CPDA128282/12), Principal investigator: Giann Barcaccia. We also thank Mirko Volpato, DAFNAE - University of Padova (Italy), for his technical help with the Real-Time RT-qPCR experiments.

\section{Author details}

${ }^{1}$ Laboratory of Plant Genetics and Genomics, DAFNAE - University of Padova, Campus of Agripolis, Viale dell'Università 16, 35020 Legnaro, Italy. ${ }^{2}$ Max Planck Institute for Chemical Ecology, Hans-Knöll-Straße 8, 07745 Jena, Germany. ${ }^{3}$ Apomixis Research Group; Department of Cytogenetics and Genome Analysis. Leibniz Institute of Plant Genetics and Crop Plant Research (IPK), Corrensstraße 3, 06466 Gatersleben, Germany.

Received: 27 November 2014 Accepted: 6 March 2015

Published online: 31 March 2015

\section{References}

1. Soltis DE, Chanderbali AS, Kim S, Buzgo M, Soltis PS. The ABC model and its applicability to basal angiosperms. Annals Botany. 2007;100:155-63.

2. Christensen CA, King EJ, Jordan JR, Drews GN. Megagametogenesis in Arabidopsis wild type and the Gf mutant. Sex Plant Reprod. 1997;10:49-64.

3. Drews GN, Lee D, Christensen CA. Genetic control of female gametophyte development and function. Plant Cell. 1998;10:1-15.

4. Johnston AJ, Meier P, Gheyselinck J, Wuest SE, Federer M, Schlagenhauf E, et al. Genetic subtraction profiling identifies genes essential for Arabidopsis reproduction and reveals interaction between the female gametophyte and the maternal sporophyte. Genome Biol. 2007;8:R204.

5. Drews GN, Koltunow AM. The female gametophyte. Arabidopsis Book. 2011;9:e0155.

6. Siddiqi I, Ganesh G, Grossniklaus U, Subbiah V. The dyad gene is required for progression through female meiosis in Arabidopsis. Development. 2000;127:197-207.

7. Gross-Hardt R, Kagi C, Baumann N, Moore JM, Baskar R, Gagliano WB, et al. LACHESIS restricts gametic cell fate in the female gametophyte of Arabidopsis. PLoS Biol. 2007:5:e47.

8. Xiadong S, Shantharaj D, Kangand X, Ni M. Transcriptional and hormonal signaling control of Arabidopsis seed development. Current Opinion Plant Biology. 2010;13:611-20.

9. Bencivenga S, Colombo L, Masiero S. Cross talk between the sporophyte and the megagametophyte during ovule development. Sex Plant Reprod. 2011;24:113-21.

10. Grossniklaus U, Schneitz K. The molecular and genetic basis of ovule and megagametophyte development. Seminars Cell Developmental Biology. 1998:9:227-38

11. Mercier R, Grelon M. Meiosis in plants: ten years of gene discovery. Cytogenetic Genome Research. 2008:120:281-90.

12. Armenta-Medina A, Demesa-Arévalo E, Vielle-Calzada JP. Epigenetic contro of cell specification during female gametogenesis. Sex Plant Reprod. 2011;24:137-47.

13. Tucker MR, Okada T, Hu Y, Scholefield A, Taylor JM, Koltunow AM. Somatic small RNA pathways promote the mitotic events of megagametogenesis during female reproductive development in Arabidopsis. Development. 2012;139:1399-404. 
14. Olmedo-Monfil V, Duran-Figueroa N, Arteaga-Vazquez M, Demesa-Arévalo E, Autran D, Grimanelli D, et al. Control of female gamete formation by a small RNA pathway in Arabidopsis. Nature. 2010;464:628-32.

15. Singh M, Goel S, Meeley RB, Dantec C, Parrinello H, Michaud C, et al. Production of viable gametes without meiosis in maize deficient for an ARGONAUTE protein. Plant Cell. 2011;23:443-58.

16. Matzk F, Hammer K, Schubert I. Coevolution of apomixis and genome size within the genus Hypericum. Sex Plant Reprod. 2003;16:51-8.

17. Barcaccia G, Arzenton F, Sharbel TF, Varotto S, Lucchin M, Parrini P. Genetic diversity and reproductive biology in ecotypes of the facultative apomict Hypericum perforatum L. Heredity. 2006;96:322-34.

18. Schallau A, Arzenton F, Johnston AJ, Hähnel U, Koszegi D, Blattner FR, et al. Identification and genetic analysis of the APOSPORY locus in Hypericum perforatum L. Plant J. 2010;62:773-84.

19. Galla G, Barcaccia G, Schallau A, Puente Molins M, Baoumlein H, Sharbel TF. The cytohistological basis of apospory in Hypericum perforatum L. Sex Plant Reprod. 2010;24:47-61.

20. Galla G, Volpato M, Sharbel TF, Barcaccia G. Computational identification of conserved microRNAs and their putative targets in the Hypericum perforatum L. flower transcriptome. Plant Reproduction. 2013;26:209-29.

21. Buckley YM, Briese DT, Rees M. Demography and management of the invasive plant species Hypericum perforatum L. using multi-level mixedeffects models for characterizing growth, survival and fecundity in a longterm data set. Journal Applied Ecology. 2003;40:481-93.

22. Nürk NM, Madriñán S, Carine MA, Chase MW, Blattner FR. Molecular phylogenetics and morphological evolution of St. John's wort (Hypericum; Hypericaceae). Molecular Phylogenetics Evolution. 2013;66:1-16.

23. Hofrichter J, Krohn M, Schumacher T, Lange C, Feistel B, Walbroel B, et al. Reduced Alzheimer's disease pathology by St. John's wort treatment is independent of hyperforin and facilitated by ABCC1 and microglia activation in mice. Curr Alzheimer Res. 2013;10:1057-69.

24. He M, Wang Y, Hua W, Zhang Y, Wang Z. De novo sequencing of Hypericum perforatum transcriptome to identify potential genes involved in the biosynthesis of active metabolites. PLoS One. 2012;7:e42081.

25. Nogler GA. Gametophytic apomixis. In: Johri BM, editor. Embryology of Angiosperms. Berlin: Springer; 1984. p. 475-518.

26. Barcaccia G, Baumlein H, Sharbel TF. Apomixis in St. John's wort: An overview and glimpse towards the future. In: Hörandle E, Grossniklaus U, Van Dijk P, Sharbel TF, editors. Apomixis: Evolution, Mechanisms and Perspectives. Vienna: Intl Association of Plant Taxonomy - Koeltz Scientific Books; 2007. p. 259-80.

27. Pupilli F, Barcaccia G. Cloning plants by seeds: Inheritance models and candidate genes to increase fundamental knowledge for engineering apomixis in sexual crops. J Biotechnology. 2012;159:291-311.

28. Koch MA, Scheriau C, Betzin A, Hohmann N, Sharbel TF. Evolution of cryptic gene pools in Hypericum perforatum: the influence of reproductive system and gene flow. Annals Botany. 2013;111:1083-94

29. Puente Molins M, Corral JM, Aliyu OM, Koch MA, Betzin A, Maron JL, et al. Biogeographic variation in genetic variability, apomixis expression and ploidy of St. John's wort (Hypericum perforatum L.) across its native and introduced range. Annals Botany. 2014;113:417-27.

30. Grimanelli D, García M, Kaszas E, Perotti E, Leblanc O. Heterochronic expression of sexual reproductive programs during apomictic development in Tripsacum. Genetics. 2003;165:1521-31.

31. Matzk F, Meister A, Brutovskà R, Schubert I. Reconstruction of reproductive diversity in Hypericum perforatum L. opens novel strategies to manage apomixis. Plant J. 2001;26:275-82.

32. Borges F, Gomes G, Gardner R, Moreno N, McCormick S, Feijó JA, et al. Comparative transcriptomics of Arabidopsis sperm cells. Plant Physiol. 2008;148:1168-81.

33. Schmidt A, Wuest SE, Vijverberg K, Baroux C, Kleen D, Grossniklaus U Transcriptome analysis of the Arabidopsis megaspore mother cell uncovers the importance of RNA helicases for plant germline development. PLoS Biol. 2011;9:e1001155

34. Schmid MW, Schmidt A, Klostermeier UC, Barann M, Rosenstiel P, Grossniklaus U. A powerful method for transcriptional profiling of specific cell types in eukaryotes: laser-assisted microdissection and RNA sequencing. PLoS One. 2012;7:e29685.

35. Pellino M, Hojsgaard D, Schmutzer T, Scholz U, Hörandl E, Vogel H, et al. Asexual genome evolution in the apomictic Ranunculus auricomus complex: examining the effects of hybridization and mutation accumulation. Mol Ecol. 2013;22:5908-21.
36. Kress WJ, Wurdack KJ, Zimmer EA, Weigt LA, Janzen DH. Use of DNA barcodes to identify flowering plants. Proc Natl Acad Sci U S A. 2005;102:8369-74.

37. Ebel C, Mariconti L, Gruissem W. Plant retinoblastoma homologues control nuclear proliferation in the female gametophyte. Nature. 2004;429:776-80.

38. Pagnussat GC, Yu HJ, Ngo QA, Rajani S, Mayalagu S, Johnson CS, et al. Genetic and molecular identification of genes required for female gametophyte development and function in Arabidopsis. Development. 2005;132:603-14

39. Demesa-Arévalo E, Vielle-Calzada JP. The classical arabinogalactan protein AGP18 mediates megaspore selection in Arabidopsis. Plant Cell. 2013;25:1274-87.

40. Moll C, Von Lyncker L, Zimmermann S, Kägi C, Baumann N, Twell D, et al. $\mathrm{CLO} / \mathrm{GFA} 1$ and ATO are novel regulators of gametic cell fate in plants. Plant J. 2008;56:913-21.

41. Kasahara RD, Portereiko MF, Sandaklie-Nikolova L, Rabiger DS, Drews GN. MYB98 is required for pollen tube guidance and synergid cell differentiation in Arabidopsis. Plant Cell. 2005;17:2981-92.

42. Portereiko MF, Lloyd A, Steffen JG, Punwani JA, Otsuga D, Drews GN. AGL80 is required for central cell and endosperm development in Arabidopsis. Plant Cell. 2006;18:1862-72.

43. Galitski T, Saldanha AJ, Styles CA, Lander ES, Fink GR. Ploidy regulation of gene expression. Science. 1999;285:251-4.

44. Albertini E, Marconi G, Reale L, Barcaccia G, Porceddu A, Ferranti F, et al. APOSTART and SERK: candidate genes for apomixis in Poa pratensis L. Plant Physiol. 2005;138:2185-99.

45. Schmidt A, Wöhrmann HJ, Raissig MT, Arand J, Gheyselinck J, Gagliardini V, et al. The Polycomb group protein MEDEA and the DNA methyltransferase MET1 interact to repress autonomous endosperm development in Arabidopsis. Plant J. 2013;73:776-87.

46. Schoft VK, Chumak N, Choi Y, Hannon M, Garcia-Aguilar M, Machlicova A, et al. Function of the DEMETER DNA glycosylase in the Arabidopsis thaliana male gametophyte. Proc Natl Acad Sci U S A. 2011;108:8042-7.

47. Garcia-Aguilar M, Michaud C, Leblanc O, Grimanelli D. Inactivation of a DNA methylation pathway in maize reproductive organs results in apomixis-like phenotypes. Plant Cell. 2010;22:3249-67.

48. Albertini E, Barcaccia G, Mazzucato A, Sharbel TF. Apomixis in the era of biotechnology. In: Pua EC, Davey MR, editors. Plant Developmental Biology Biotechnological Perspectives. Berlin Heidelberg: Springer; 2010. p. 405-36.

49. Albertini E, Marconi G, Barcaccia G, Raggi L, Falcinelli M. Isolation of candidate genes for apomixis in Poa pratensis L. Plant Mol Biol. 2004;56:879-94.

50. Barcaccia G, Albertini E. Apomixis in plant reproduction: a novel perspective on an old dilemma. Plant Reproduction. 2013:26:159-79.

51. Sharbel TF, Voigt ML, Corral JM, Galla G, Kumlehna J, Klukasa C, et al. Apomictic and sexual ovules of Boechera display heterochronic global gene expression patterns. Plant Cell. 2010;22:655-71

52. Tucker MR, Araujo AC, Paech NA, Paech NA, Hecht V, Schmidt EDL, et al. Sexual and apomictic reproduction in Hieracium subgenus pilosella are closely interrelated developmental pathways. Plant Cell. 2003;15:1524-37.

53. Galla G, Zenoni S, Marconi G, Marino G, Botton A, Pinosa F, et al. Sporophytic and gametophytic functions of the cell cycle- associated Mob1 gene in Arabidopsis thaliana L. Gene. 2011;484:1-12.

54. Zhu YY, Machleder EM, Chenchik A, Li R, Siebert PD. Reverse transcriptase template switching: a SMART approach for full-length cDNA library construction. Biotechniques. 2001;30:892-7.

55. Zhulidov PA, Bogdanova EA, Shcheglov AS, Vagner LL, Khaspekov GL, Kozhemyako VB, et al. Simple cDNA normalization using kamchatka crab duplex-specific nuclease. Nucleic Acids Res. 2004;32:e37.

56. Vogel H, Altincicek B, Glöckner G, Vilcinskas A. A comprehensive transcriptome and immune- gene repertoire of the lepidopteran model host Galleria mellonella. BMC Genomics. 2011;12:308.

57. Vogel $\mathrm{H}$, Wheat $\mathrm{CW}$. Accessing the Transcriptome: How to Normalize mRNA Pools. Methods Molecular Biology. 2011;772:105-28.

58. Oliveros JC. VENNY. An interactive tool for comparing lists with Venn Diagrams. 2007, http://bioinfogp.cnb.csic.es/tools/venny/index.html.

59. Rozas J, Rozas R. DnaSP version 3: an integrated program for molecular population genetics and molecular evolution analysis. Bioinformatics. 1999:15:174-5

60. Conesa A, Götz S, García-Gómez JM, Terol J, Talón M, Robles M. Blast2GO: a universal tool for annotation, visualization and analysis in functional genomics research. Bioinformatics. 2005;21:3674-6. 
61. Galla G, Barcaccia G, Ramina A, Collani S, Alagna F, Baldoni L, et al.

Computational annotation of genes differentially expressed along olive fruit development. BMC Plant Biol. 2009;9:128.

62. Pfaffl MW. A new mathematical model for relative quantification in real-time RT-PCR. Nucleic Acids Res. 2001;29:2002-7.

63. Usadel B, Poree F, Nagel A, Lohse M, Czedik-Eysenberg A, Stitt M. A quide to using MapMan to visualize and compare Omics data in plants: a case study in the crop species, Maize. Plant Cell Environment. 2009;32:1211-29.

Submit your next manuscript to BioMed Central and take full advantage of:

- Convenient online submission

- Thorough peer review

- No space constraints or color figure charges

- Immediate publication on acceptance

- Inclusion in PubMed, CAS, Scopus and Google Scholar

- Research which is freely available for redistribution 